ARTICLE

https://doi.org/10.1038/s41467-019-13237-8

\title{
Glycogen branching enzyme controls cellular iron homeostasis via Iron Regulatory Protein 1 and mitoNEET
}

Nhan Huynh1, Qiuxiang Ou, Pendleton Cox ${ }^{1}$, Roland Lill (i) ${ }^{2,3}$ \& Kirst King-Jones (iD ${ }^{1 *}$

Iron Regulatory Protein 1 (IRP1) is a bifunctional cytosolic iron sensor. When iron levels are normal, IRP1 harbours an iron-sulphur cluster (holo-IRP1), an enzyme with aconitase activity. When iron levels fall, IRP1 loses the cluster (apo-IRP1) and binds to iron-responsive elements (IREs) in messenger RNAs (mRNAs) encoding proteins involved in cellular iron uptake, distribution, and storage. Here we show that mutations in the Drosophila 1,4-Alpha-Glucan Branching Enzyme ( $A G B E$ ) gene cause porphyria. AGBE was hitherto only linked to glycogen metabolism and a fatal human disorder known as glycogen storage disease type IV. AGBE binds specifically to holo-IRP1 and to mitoNEET, a protein capable of repairing IRP1 ironsulphur clusters. This interaction ensures nuclear translocation of holo-IRP1 and downregulation of iron-dependent processes, demonstrating that holo-IRP1 functions not just as an aconitase, but throttles target gene expression in anticipation of declining iron requirements.

\footnotetext{
${ }^{1}$ Department of Biological Sciences, University of Alberta, G-504 Biological Sciences Bldg, Edmonton, Alberta T6G 2E9, Canada. ${ }^{2}$ Institut für Zytobiologie und Zytopathologie, Philipps-Universität Marburg, Robert-Koch-Strasse 6, 35032 Marburg, Germany. ${ }^{3}$ LOEWE Zentrum für Synthetische Mikrobiologie SynMikro, Philipps-Universität Marburg, Hans-Meerwein-Straße, 35043 Marburg, Germany. *email: kirst.king-jones@ualberta.ca
} 
ron is an essential trace element for nearly all organisms and cells, because iron co-factors, most commonly in the form of haem and iron-sulfur $(\mathrm{Fe}-\mathrm{S})$ clusters $^{1,2}$, are required for a wide range of protein functions. While essential, free iron is also highly reactive and cytotoxic, and thus its acquisition requires tight regulation by cells ${ }^{2}$. IRP1 is a bifunctional protein because it can reversibly bind to $\mathrm{Fe}-\mathrm{S}$ clusters ${ }^{3}$. Under iron-replete conditions, the protein forms holo-IRP1 and acts as a cytosolic aconitase, an enzyme that interconverts citrate and isocitrate. When cellular iron levels drop, IRP1 loses its Fe-S cluster and assumes a different conformation ${ }^{4}$, apo-IRP1, which then binds to and regulates specific target $\mathrm{mRNAs}$ containing iron-responsive elements (IREs). This action can either block translation or enhance transcript stability, depending on the location of the IRE, with a net outcome that promotes increased cellular iron availability and trafficking 5 .

Current models for studying cellular iron homeostasis are limited in the sense that they have either static, or at best, linearly increasing iron requirements (cell cultures and developing erythrocytes, respectively) ${ }^{2,6}$. We introduce here the Drosophila prothoracic gland (PG) as a model to study highly dynamic iron requirements. The PG is an endocrine tissue mainly devoted to the production of steroid hormones in developing insects. In both vertebrates and insects, the synthesis of steroid hormones is largely dependent on enzymes that require haem and $\mathrm{Fe}-\mathrm{S}$ clusters $^{7,8}$. In Drosophila, the PG is the principal steroidproducing gland, and part of a larger endocrine tissue called the ring gland. During larval development, the PG produces pulses of the steroid hormone ecdysone (E), which acts as a recurring systemic signal that controls gene expression in target tissues to coordinate hatching, moulting, and metamorphosis (Supplementary Fig. 1). The first step of E biosynthesis is carried out by Neverland, which harbours an Fe-S cluster ${ }^{9}$, while all but one of the following reactions are carried out by cytochrome P450 proteins, which require haem as an obligate co-factor (Fig. 1a). The last larval stage of Drosophila development is accompanied by exceedingly high expression of ecdysone-producing enzyme ("Halloween") genes (Supplementary Fig. 1) ${ }^{8}$, indicating that the PG requires substantial amounts of iron, which can be visualized by staining for ferric iron (Fig. 1b). PG cells have fluctuating iron and haem demands because they must match the rise and fall of Halloween enzyme levels with appropriate production rates of iron co-factors. Thus, the PG represents a powerful model to study mechanisms by which cells control dynamic changes in cellular iron and haem requirements. We show here that the Drosophila Glycogen Branching Enzyme (AGBE) is a regulator of iron homeostasis. AGBE interacts physically with the holoform of Iron Regulatory Protein 1A (IRP1A) and Cisd2, an ortholog of vertebrate mitoNEET. This synergistic interaction ensures that holo-IRP1A remains functional. Further, we show that holoIRP1A has a surprising new role in the nucleus, where it transcriptionally downregulates genes acting in steroid hormone biosynthesis as well as iron and heme metabolism.

\section{Results}

Loss of glycogen branching enzyme causes porphyria-like phenotypes. The haem biosynthetic pathway is highly conserved in metazoans and fungi and comprises eight enzymatic steps that convert glycine and Succinyl-CoA to mature haem (Fig. 1c) $)^{10}$. Exposure to air and UV light isomerizes porphyrinogen rings, first produced in step 4, into autofluorescening porphyrins, but incorporation of iron into protoporphyrin IX results in nonfluorescing haem (Fig. 1d) ${ }^{10,11}$. We noticed the presence of red autofluorescence in the PG when we exposed larvae from four RNAi lines to UV light (targeting Updo, Ppox, spz5, and AGBE), all of which had been identified in two unrelated PG-specific RNA interference (RNAi) screens ${ }^{7,8}$. Also, the ring glands were enlarged compared to time-matched controls and had a redbrown appearance under brightfield light (Supplementary Fig. 2). A fifth RNAi line, Nos ${ }^{I R-X}$, targeting the nitric oxide synthase (Nos) gene, had been reported to produce large red-brown PGs ${ }^{12}$, and when re-examined by us, also showed red autofluorescence (Fig. 1e). Consistent with their role in haem biosynthesis, depleting Updo (=vertebrate UROD, Supplementary Fig. 3) and Ppox caused protoporphyrin accumulation in the PG. This is equivalent to what occurs in patients afflicted with porphyria, a group of rare diseases impairing haem biosynthesis ${ }^{13}$. PG-specific Alas-RNAi, on the other hand, disrupts haem synthesis prior to porphyrinogen ring formation, and therefore lacked the autofluorescence, but causes enlarged ring glands (Fig. 1e). We then sought to validate the three remaining lines, Nos ${ }^{I R-X}, A G B E^{I R 1}$, and $s p z 5^{I R}$, since their relationship to haem biosynthesis was intriguing. We were unsuccessful in finding independent evidence for the $\operatorname{Nos}^{I R-X}$ and $s p z 5^{I R}$ lines, suggesting that the phenotypes were caused by off-target effects. However, a second, nonoverlapping RNAi line targeting $A G B E, A G B E^{I R 2}$, caused similar phenotypes (Fig. 1f). $A G B E$ encodes a glycogen branching enzyme, which is an essential enzyme that acts in glycogen biosynthesis ${ }^{14}$. There are, however, no reports that link glycogen branching enzymes to iron or haem homeostasis. Therefore, we further validated these results by using CRISPR/CAS9 to replace the endogenous $A G B E$ gene with a genomic copy that was flanked by Flippase (FLP) Recombinase Target (FRT) sites, and where the last exon extended into a Flag- or Myc-tag $\left(A G B E^{F C F}\right.$ and $A G B E^{F C M}$, Supplementary Fig. 4). Excision of the conditional $A G B E^{F C F}$ allele via PG-specific expression of FLP confirmed the $A G B E-$ RNAi phenotypes, as we observed strong autofluorescence with overall higher penetrance than the two RNAi lines, since no adults eclosed compared to $4.6 \%$ in homozygous $P G>A G B E^{I R 1}$ animals (Fig. 1f, g).

We reasoned that a lack of cellular or mitochondrial iron could disrupt haem production and may explain the porphyria phenotype in $A G B E$-loss-of-function lines. Remarkably, upon rearing $P G>A G B E^{I R 1}$ and $P G>F L P ; A G B E^{F C F}$ larvae on an iron-supplemented diet, the autofluorescence was absent (Fig. 1f), and we observed that $\sim 40-50 \%$ of the larvae now developed into phenotypically normal adults (Fig. 1g). In agreement with this, adding the iron chelator bathophenanthroline sulfate (BPS) slightly decreased survival rates, while adding both iron and BPS to the diet reversed the rescue seen by iron alone, confirming that BPS is an effective tool to reduce available iron. AGBE-RNAi larvae were uniquely rescued by dietary iron, since neither Alas-, Updo-, Ppox-, Nos- or spz5-RNAi lines benefited from iron supplementation (not shown). AGBE expression was moderately upregulated under iron-chelating conditions, consistent with the idea that the gene partakes in cellular iron homeostasis (Fig. 1h). Next, we tested whether disrupting four other glycogen biosynthesis genes via PG-specific RNAi would phenocopy AGBE-depletion (Supplementary Fig. 5). This neither caused autofluorescence nor significant lethality. However, ubiquitous expression of RNAi targeting these glycogen biosynthesis genes caused widespread larval lethality, confirming that all RNAi transgenes in these lines were expressed, which suggested that the disruption of glycogen biosynthesis per se in the PG did not cause any iron- or haem-related phenotypes, but was a unique feature of AGBE.

Glycogen branching enzyme physically interacts with Iron Regulatory Protein 1 (IRP1). AGBE is the single orthologue of vertebrate GBE1 (Glycogen Branching Enzyme 1), and the two 


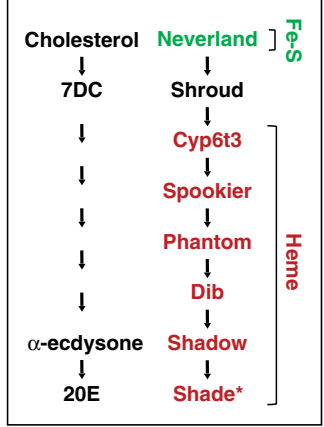

b

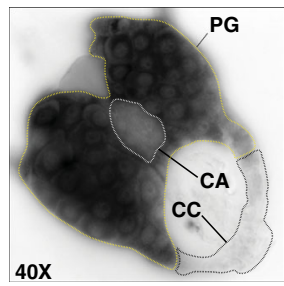

$40 X$

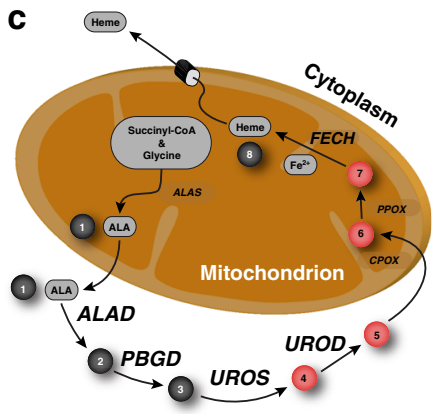

g

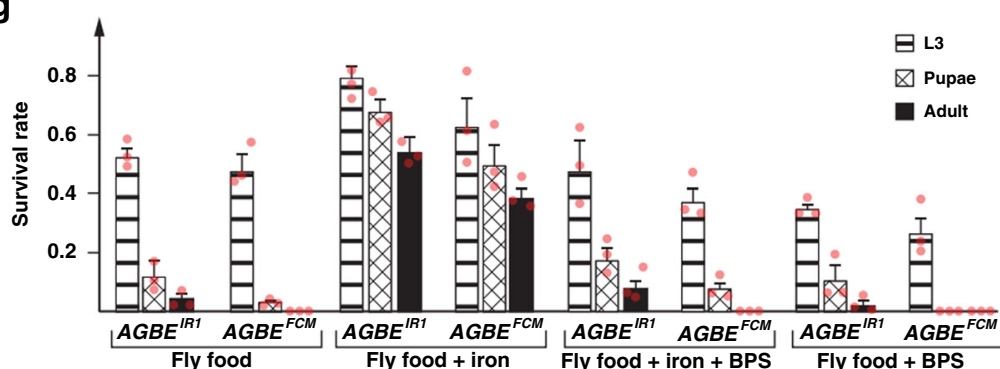

d

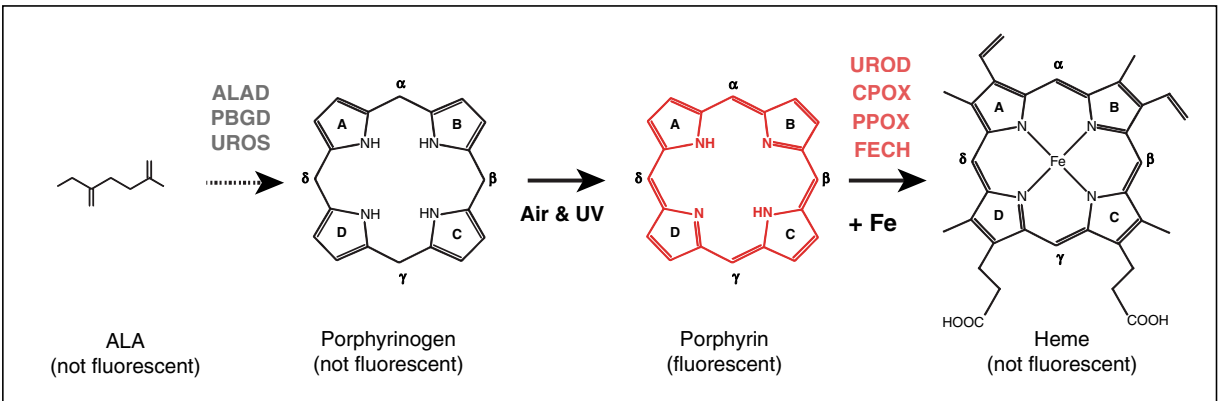

e

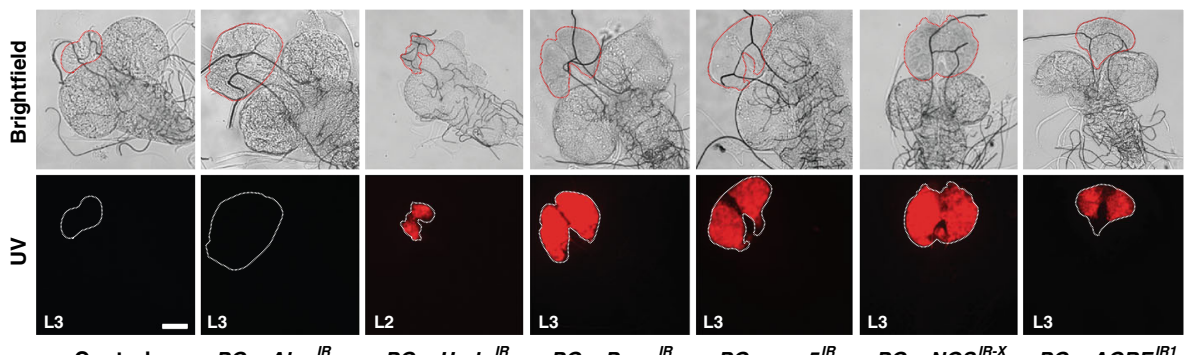

f

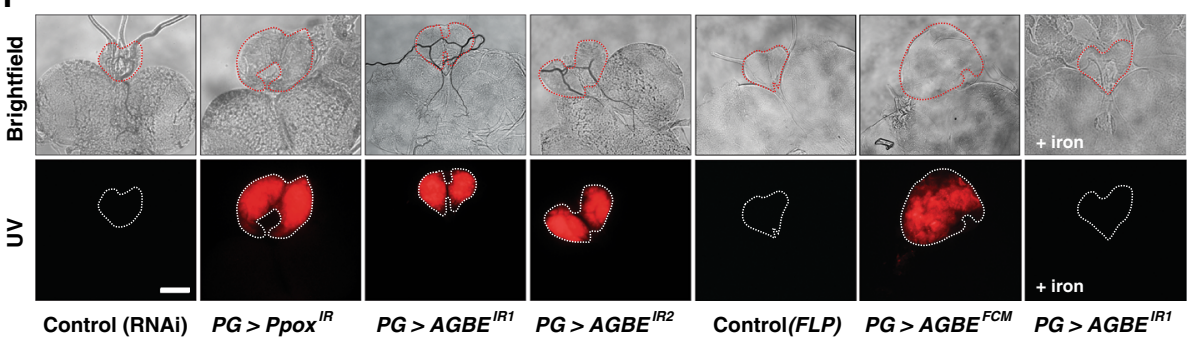

Fig. 1 Disruption of haem biosynthesis in the Drosophila prothoracic gland (PG). a Ecdysone biosynthetic pathway converts cholesterol to $\alpha$-ecdysone, which is metabolized to $200 \mathrm{H}$-ecdysone in target cells by Shade ( ${ }^{\star}$ not or lowly expressed in the PG). All enzymes except for Shroud require iron co-factors in the form of iron-sulfur (Fe-S) clusters or haem. b Stain for ferric (non-haem-bound) iron in the ring gland. The corpus allatum (CA) and the corpora cardiaca (CC) are neighbouring glands fused to the PG. c Haem biosynthesis pathway in metazoans and yeast. Red circles represent protoporphyrin intermediates that autofluoresce. d Autofluorescence of porphyrins occurs through isomerization of porphyrinogens exposed to air and UV light. e UV exposure of dissected ring glands from RNAi lines (designated as gene ${ }^{\mathrm{R}}$ ) from second (L2) or third (L3) instar stages. Alas, Updo, and Ppox encode haemsynthesizing enzymes. spz5: spaetzle5, Nos: nitric oxide synthase, AGBE: 1,4-Alpha-Glucan Branching Enzyme. Scale bar $=250 \mu \mathrm{m}$. $\mathbf{f} U V$ exposure of dissected ring glands isolated at $40 \mathrm{~h}$ after the $\mathrm{L} 2 / \mathrm{L} 3$ moult ( $~ 8 \mathrm{~h}$ prior to pupariation in controls). RNAi lines $A G B E^{I R 1}$ and $A G B E^{I R 2}$ target distinct regions of the AGBE mRNA. AGBEFCM is a conditional CRISPR-knock-in allele that can be excised in a tissue-specific manner via the expression of Flippase (FLP) recombinase (Supplementary Fig. 4). + iron: larvae were reared on a diet containing ferric ammonium citrate (FAC) as an iron supplement. Scale bar $=250 \mu \mathrm{m}$. $\mathbf{g}$ Survival of $A G B E^{I R 1}$ and $A G B E^{F C M}$ larvae fly food supplemented with iron (FAC) or an iron chelator, bathophenanthroline sulfate (BPS). Error bars represent standard deviation. Three biological replicates, with each sample containing 50 individuals. $\mathbf{h}$ Relative AGBE mRNA expression levels. Dissected ring glands: isolated from L3 reared on media \pm BPS. Cultured ring glands: isolated from L3 reared on normal media, but then transferred to buffer containing \pm BPS. S2 cells: Schneider 2 cells grown on medium \pm BPS. mRNA levels were analysed via quantitative real-time PCR. For primers see Table 3 . Asterisk indicates a $P$-value $<0.05$ based on the Student's $t$ test. Error bars represent $95 \%$ confidence intervals. Each of the three biological replicates was tested three times. Source data are provided as a Source Data file. 
proteins are $61 \%$ identical. A search of protein-protein interaction databases ${ }^{15,16}$ revealed that human GBE1 physically interacts with IRP1. Vertebrates have two IRP genes, IRP1 and IRP2, but only IRP1 can switch between the aconitase and the RNA-binding form, while IRP2 lacks the Fe-S cluster and is constitutively RNA-binding ${ }^{3}$. Drosophila does not have the IRP2 gene but harbours two IRP1 genes (IRP1A and IRP1B) (Supplementary Fig. 6). Only IRP1A has been shown to switch from holo- to the IRE-binding apoform, while IRP1B is believed to act only as an aconitase, as it failed to bind canonical IREs ${ }^{17}$. The reported interaction between GBE1 and IRP1 raised the possibility that Drosophila AGBE and human GBE1 function in the regulation of iron homeostasis by modulating IRP1 activity. Using a cell culture approach, we established that the interaction also occurred in Drosophila, namely between AGBE and IRP1A (Fig. 2a), and we recapitulated the interaction between human IRP1 and GBE1 in the same system (Fig. 2b). AGBE interacted robustly with wild type IRP1A, as well as with IRP1A 3 R3Q, which carries three point mutations (R549Q, R554Q, R793Q, see Supplementary Fig. 4) predicted to disrupt RNA-binding ${ }^{18}$. Strikingly, a single point mutation that prevents $\mathrm{Fe}-\mathrm{S}$ cluster binding to IRP1A (predicted to generate constitutively RNA-binding IRP1A ${ }^{\mathrm{C} 450 \mathrm{~S}}$ ) abolished the interaction with AGBE (Fig. 2a), suggesting that holo-IRP1A is the in vivo binding partner of AGBE. This was paradoxical, as $A G B E$ mutations caused iron-deficiency phenotypes, but holoIRP1A has no known roles in iron homeostasis as it is believed to only act as an aconitase. To examine whether the IRP $1 \mathrm{~A}^{3 \mathrm{R} 3 \mathrm{Q}}$ and IRP1A $\mathrm{A}^{\mathrm{C} 450 \mathrm{~S}}$ forms acted as predicted, we tested their in vivo ability to bind an IRE-containing mRNA $(S d h B)$ and whether either of them had aconitase activity (Fig. 2c, d). As expected, the IRP1A $3 R 3 Q$ form displayed strongly reduced mRNA-binding compared to both the wild type and the IRP $1 \mathrm{~A}^{\mathrm{C} 450 \mathrm{~S}}$ forms (Fig. 2c). Likewise, both knocked-in and transgenic alleles of $I R P 1 A^{C 450 S}$ resulted in significantly reduced aconitase activity compared to wild type IRP1A and the IRP1A $\mathrm{A}^{3 \mathrm{R} 3 \mathrm{Q}}$ form (Fig. $2 \mathrm{~d}$ and Supplementary Fig. 7).

To further substantiate the interaction between AGBE and holo-IRP1A, we carried out a series of MALDI-TOF-based mass spectrometry (MS) experiments (see later section). We also sought to validate this interaction by genetic means. For the latter, we tested whether animals with PG-specific loss of $A G B E$ function could be rescued by expressing transgenic wild type $I R P 1 A$ or IRP1A C450S (Supplementary Table 1). Remarkably, wild type IRP1A rescued both the larval lethality (Fig. 2e, $\mathrm{f}$ ) as well as the porphyria phenotype of animals that lacked functional AGBE (Fig. 2g), while IRP1A C450S was completely ineffective (Fig. 2e, g). It was possible that the IRP $1 A^{C 450 S}$ allele was not functional, despite differing only in a single point mutation from IRPIA. However, when we expressed IRP1A ${ }^{C 450 S}$ in other genetic backgrounds, we observed dramatic rescue of $P G>N O S^{I R-X}$ RNAi animals with respect to both the lethality (Fig. 2f) and protoporphyrin accumulation (not shown). Also, our immunoprecipitation results showed that IRP1AC450S is RNA-binding (Fig. 2c). Taken together, these data demonstrated that IRP $1 A^{C 450 S}$ was fully active but not sufficient to compensate for the iron deficiency in AGBE-depleted animals, suggesting that holo-IRP1A has functions beyond the aconitase that are important for iron homeostasis. To test whether the aconitase function of holo-IRP1A had unexpected essential functions, we attempted rescuing $A G B E^{F C M}$ mutants with the aconitase-only form of IRP1A (IRP1A ${ }^{3 R 3 Q}$ ), as well as a cytosolic and mitochondrial version of yeast aconitase (YAcol ${ }^{W T}$ and $Y A c o 1^{\Delta S p}$, respectively), neither of which can switch to the RNA-binding form ${ }^{18,19}$. None of these approaches rescued the loss-of-AGBE-function phenotypes (Fig. $2 \mathrm{~g}$ ), indicating that both holo- and apo-IRP1A were required for survival. Lastly, we crossed human IRP1 (hIRP1) and IRP2 (hIRP2) into the $A G B E^{I R 1}$-RNAi strain. Consistent with the above findings, only hIRP1 (equivalent to IRP1A, Supplementary Fig. 6) could fully rescue $A G B E^{I R 1}$ larvae, while constitutively RNA-binding hIRP2, was much less effective (Fig. 2g), albeit more efficient than IRP1A ${ }^{C 450 S}$, suggesting partial rescue by hIRP2.

Since no null mutations were available for IRP $1 A$ or $I R P 1 B$, we needed to establish that a) these genes had indeed roles in Drosophila iron regulation and if so, b) whether IRP1A and IRP1B had distinct roles in controlling cellular iron levels, c) whether these genes were required in the PG, and d) whether this would phenocopy AGBE-depletion in the PG. We first disrupted both $I R P 1 A$ and $I R P 1 B$ in the $P G$ via RNAi $\left(P G>I R P 1 A^{I R}\right.$ and $P G>$ $\left.I R P 1 B^{I R}\right)$. On regular fly food, neither RNAi line resulted in obvious phenotypes. However, when flies were reared on irondepleted fly food for three generations, $P G>I R P 1 A^{I R}$ animals displayed significant larval lethality, with a concomitant appearance of red autofluorescence in the larval PG (Fig. 3a). Control and $P G>I R P 1 B^{I R}$ populations did not exhibit lethality until the 5th generation, and larvae never showed any autofluorescence (not shown and Supplementary Fig. 8). To confirm these data, we used two approaches. First, we generated a Flag-tagged and FRTflanked knock-in allele of endogenous IRP1A (IRP1A FCF), allowing us to excise the gene via PG-specific expression of FLP (Supplementary Fig. 4). This approach resulted in red-fluorescing PGs in PG $>$ FLP;IRP1A FCF larvae that were switched from ironreplete to BPS-containing media (Fig. 3b). However, homozygous $I R P 1 A^{F C F}$ flies were not viable on regular fly media, indicating that the inserted FRT sites had generated a loss-of-IRP1Afunction allele. Therefore, we employed a second CRISPR strategy, where we crossed flies that specifically expressed CAS9 in the $\mathrm{PG}^{20}$ to flies expressing two gRNAs that targeted IRP1A (Supplementary Table 1). The resulting F1 progeny also displayed PG-specific autofluorescence and $100 \%$ lethality on regular fly media (Fig. 3b). To complement these PG-specific lesions with classic mutant analysis, we examined an existing mutant IRP1A line (Bloomington \#30181). However, we did not consider this allele further as it turned out to be a weak hypomorph. We, therefore, generated deletion mutants for IRP1A and IRP1B using CRISPR/CAS9, designated here as IRPIA ${ }^{K O}$ and $I R P 1 B^{K O}$. On regular fly food, $I R P 1 A^{K O}$ mutants died as first (L1) and second instar larvae (L2), but were able to develop into phenotypically normal adults when reared on an iron-supplemented diet (Fig. 3c), indicating that IRP1A was essential for responding to iron-poor conditions. In contrast, IRP1B mutants revealed no obvious phenotypes under any of the tested conditions. Taken together, these results showed that IRP1A is the principal regulator of cellular iron homeostasis in Drosophila and that IRP1A depletion phenocopied the iron-dependent porphyria seen in $A G B E$ mutants.

Subcellular localization of apo- and holo-IRP1. We then addressed whether holo-IRP1A had unanticipated roles in the regulation of cellular iron homeostasis, since only the holoform interacted with $\mathrm{AGBE}$, and was required to rescue $A G B E$ mutants. When we carried out immunolocalisation of $P G>$ $3 x$ Flag-IRP $1 A^{W T}$ and $P G>3 x$ Flag-IRP $1 B^{W T}$ transgenic lines (Supplementary Table 1), we found that both IRP1A and IRP1B were enriched in PG nuclei (Fig. 3d). In stark contrast, expressing the single-point mutation variants $I R P 1 A^{C 450 S}$ or IRP1BC447S (which abolishes Fe-S-binding in IRP1B, Supplementary Table 1) resulted in predominantly cytoplasmic accumulation of either protein (Fig. 3d). Similarly, we found that human IRP1 localises to PG nuclei as well, while human IRP2 failed to do so (Fig. 3e). This behaviour is consistent with the absence of an $\mathrm{Fe}-\mathrm{S}$ cluster in 

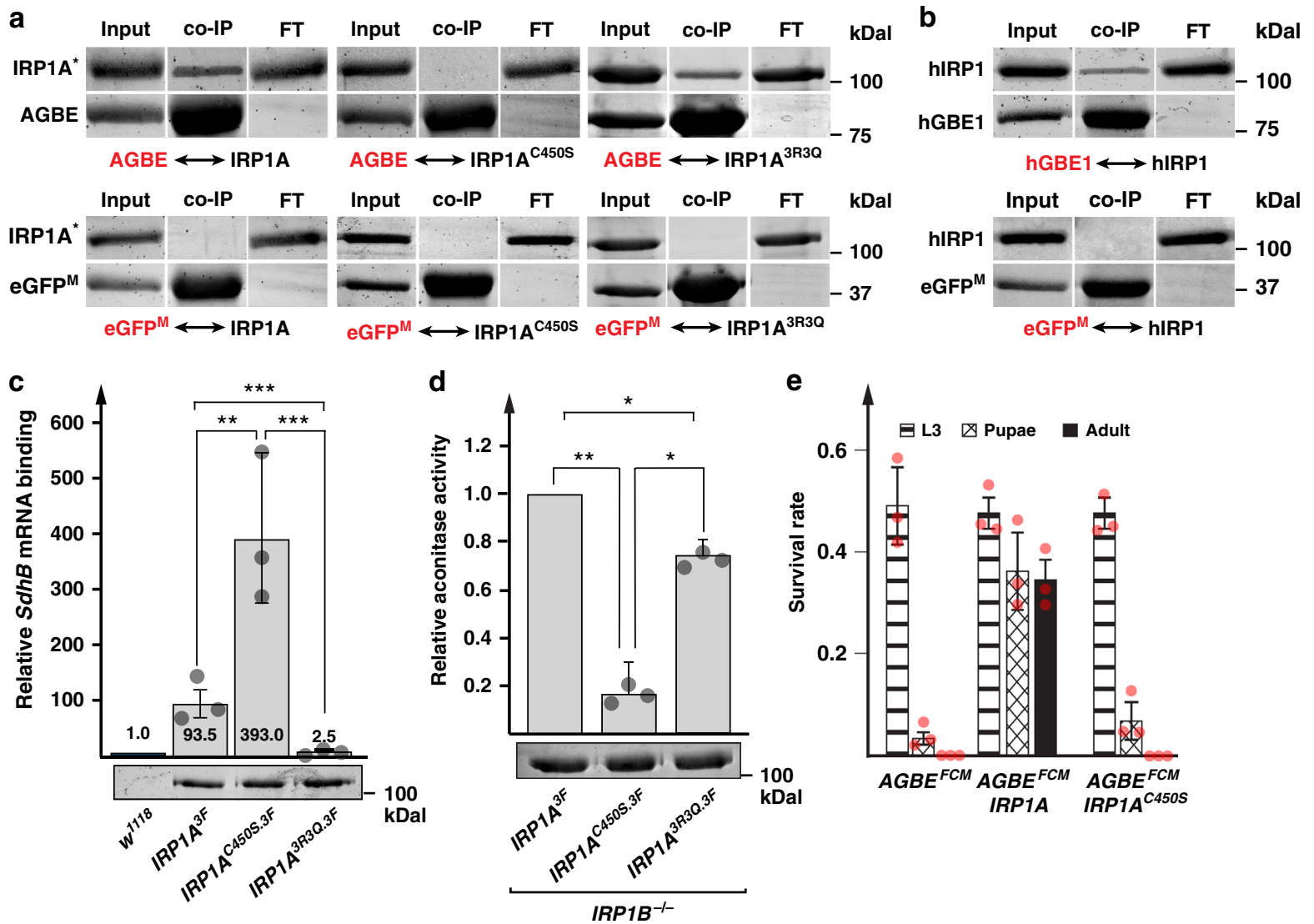

f

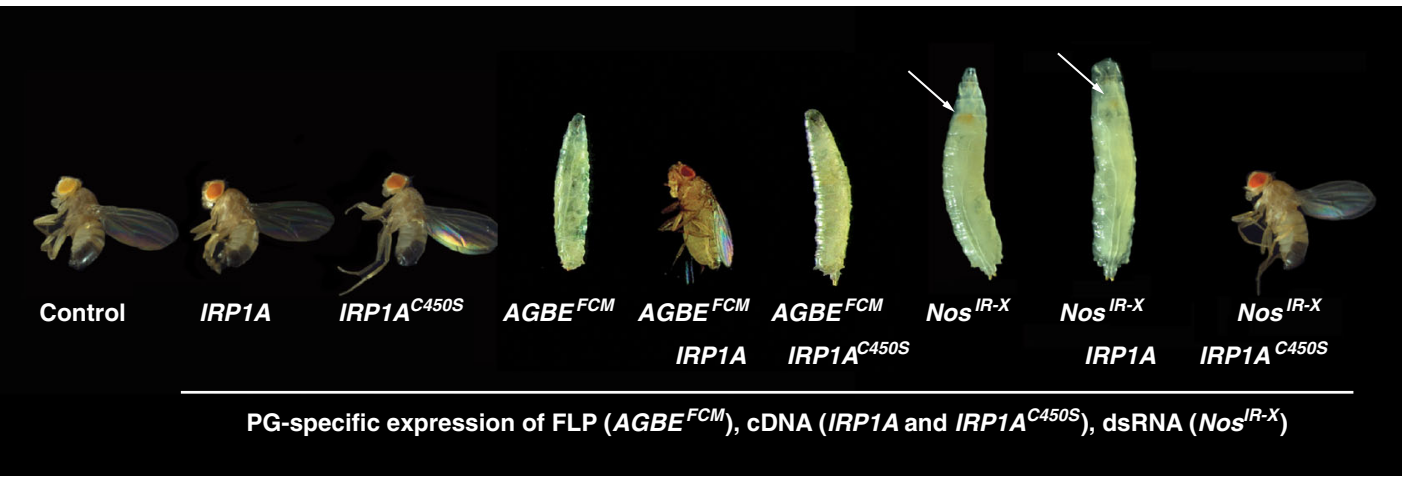

g

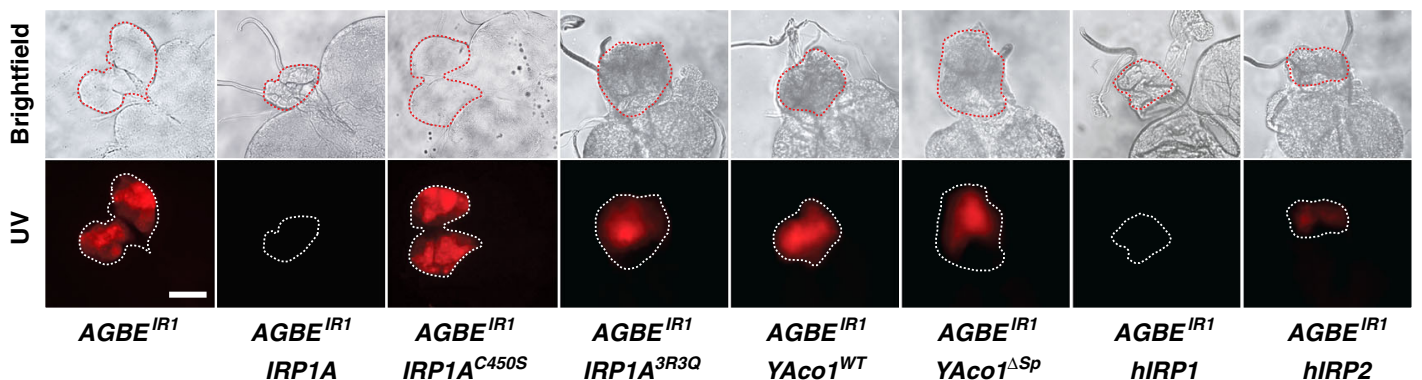

hIRP2, indicating that nuclear accumulation strongly favours holo-IRP proteins.

Given the interaction between AGBE and holo-IRP1A, we wondered whether entry of IRP1A into nuclei was dependent on AGBE. For this, we crossed the Flag-tagged $I R P 1 A^{3 F}$ and $I R P 1 B^{3 F}$ knock-in alleles (Supplementary Fig. 4) into $A G B E$ mutants. This approach revealed that IRP1A, but not IRP1B, was dependent on
$A G B E$ for nuclear translocation (Fig. 3f), suggesting that IRP1A requires $\mathrm{AGBE}$ for maintaining $\mathrm{Fe}-\mathrm{S}$ clusters, which in turn are needed for nuclear entry. Finally, we determined the subcellular localisation of IRP $1 \mathrm{~A}^{3 \mathrm{~F}}$ and IRP1B ${ }^{3 \mathrm{~F}}$ in other tissues. Both IRP1A and IRP1B showed strong cytoplasmic and negligible nuclear presence in the larval salivary gland, while the adjacent fat body displayed predominantly nuclear IRP1A and IRP1B (Fig. 3g). 
Fig. 2 AGBE interacts with IRP1A. a Co-transfection of S2 cells with plasmids encoding Flag-tagged IRP1A variants (IRP1A ${ }^{\star}$ ) and Myc-tagged AGBE followed by immunoprecipitation via anti-Myc antibodies and Western blotting. Names shown in red indicate the protein used as bait. IRP1A: wild type IRP1A, IRP1AC450S: constitutively RNA-binding IRP1A, IRP1A3R3Q: non-RNA-binding form of IRP1A (Supplementary Table 1). Myc-tagged enhanced GFP (eGFPM) served as a negative control. Input lane represents $10 \%$ of the sample. Presence of co-immunoprecipitated proteins were tested with anti-Flag antibodies. b Like A, but co-transfection of S2 cells with plasmids encoding Flag-tagged human IRP1 (aka Aco1) and Myc-tagged human GBE1, as well as eGFPM as a negative control. c Quantitative RNA-immunoprecipitation (RIP). Samples from larvae carrying Flag-tagged knock-in alleles of IRP1A (IRP1A ${ }^{3 F}$, IRP1AC450S.3F, and IRPIA $3 R 3 Q .3 F$ ) (Supplementary Fig. 4) were normalized via Western blotting to visualize Flag-tagged proteins followed by ImageJ quantification. Western blot of adjusted samples shown below graph. Untagged IRP1A (control line $w^{1118}$ ) served as a negative control and calibrator (normalized expression =1). SdhB mRNA harbours a validated IRE 72,73 . Co-immunoprecipitated SdhB mRNA was quantified via qPCR. Error bars represent $95 \%$ confidence intervals from three biological replicates. ${ }^{\star \star \star} p<0.001,{ }^{\star \star} p<0.01,{ }^{\star} p<0.05$. $\mathbf{d}$ Aconitase activity. Same IRP1A alleles and normalization procedure as described in c, except that IRPIA ${ }^{3 F}$ served as the control (normalized to 1). All alleles were crossed into an IRP1B-/- mutant background to eliminate the aconitase activity of IRP1B. Further, we removed mitochondria via ultracentrifugation to reduce the contribution of mitochondrial aconitase. Error bars represent standard deviation from three biological replicates. ${ }^{\star \star} p<0.01,{ }^{\star} p<0.05$. e Survival rates of $P G>F L P ; A G B E F C M$ animals

(Supplementary Fig. 4), which causes Flippase-mediated excision of the AGBE transcription unit specifically in the prothoracic gland (PG). Tested in either the presence or absence of the IRPIA and IRPIAC450S transgenes that are also expressed in a PG-specific manner. Error bars represent standard deviation from three biological replicates (each sample contained 50 individuals). $\mathbf{f}$ Larval and adult phenotypes of $P G>F L P ; A G B E^{F C M}$ and $P G>$ Nos $I R-X$ animals expressing IRP1A ${ }^{C 450 S}$ or wild type IRP1A transgenes. Arrows point to red-stained PG. $\mathbf{g}$ Ring glands dissected from $P G>A G B E^{I R 1}$ larvae in the presence or absence of the following transgenic CDNAs: IRP1A (wild type IRP1A); IRP1AC450S (constitutively RNA-binding); IRP1A ${ }^{3 R 3 Q}$ (non-RNA-binding); YAcolWT: wild type yeast aconitase (mitochondrial); YAco1 $\triangle S$ P (cytoplasmic); hIRP1 \& hIRP2: human IRP1 \& IRP2. Scale bar $=250 \mu \mathrm{m}$. All transgenes are expressed in a PGspecific manner via the Gal4-UAS system. Source data are provided as a Source Data file.

This strongly suggests that nuclear translocation of IRP1 proteins is highly tissue-specific, and not a function of systemic iron load, and hence may reflect tissue-specific iron requirements. Given that human IRP1 also localises to Drosophila nuclei, this raises the question of whether vertebrate IRP1 may also enter nuclei in specific tissues or during specific developmental/physiological conditions.

We next sought to identify proteins that would physically interact with IRP1A and IRP1B in order to shed light on the interaction with AGBE and the presence of both IRP1s in nuclei. For this, we immunoprecipitated endogenous or transgenic versions of Flag-tagged AGBE, IRP1A and IRP1B and subjected ring gland and whole-body samples to MS, for a total of 17 conditions (Supplementary Table 2). As controls, we used a total of five wild type samples (which lack Flag-tagged proteins), processed them in parallel to the experimental samples, and removed all proteins found in the controls from the experimental data sets (Supplementary Data 1). Briefly, the interactome for IRP1A indicated extensive interactions with ribosomal proteins and eukaryotic initiation factors, consistent with previous findings ${ }^{21,22}$ and IRP's role in regulating translation. We also identified four histone proteins for IRP1A (H4, H2A, H2B, and $\mathrm{H} 2 \mathrm{Av}$ ) and two for IRP1B ( $\mathrm{H} 4$ and $\mathrm{H} 2 \mathrm{~A})$, consistent with the presence of both IRP1s in nuclei (Fig. 4a and Supplementary Data 1-2). Importantly, endogenously tagged AGBEFCF specifically pulled down a total of 22 proteins from whole-body samples (Fig. 4b), which included IRP1A, IRP1B and Cisd2, an Fe-S protein. Vertebrate Cisd1, Cisd2, and Cisd3 comprise a small family referred to as the NEET proteins ${ }^{23}$, which harbour an unusual $2 \mathrm{Fe}-2 \mathrm{~S}$ cluster that enables these proteins to transfer their cluster to other proteins ${ }^{24-26}$. Drosophila encodes only two NEET proteins, Cisd2 and CG3420 (=> Cisd3), where Cisd2 lies evolutionary between human mitoNEET (encoded by Cisd1) and Naf-1 (encoded by Cisd2) ${ }^{27}$, and as such, fly Cisd 2 may be functionally related to both.

We also used endogenously tagged IRP1A (IRP1A ${ }^{3 \mathrm{~F}}$ ) as bait, which co-immunoprecipitated 166 proteins that included AGBE, Cisd2, and IRP1B, as well as two ferritins (iron storage proteins), Fer1HCH and Fer2LCH (Fig. 4a, b and Supplementary Data 1-2). Both AGBE and IRP1A interacted with another glycogen enzyme, glycogen synthase (GlyS), further corroborating that cellular iron homeostasis and glycogen metabolism are physically linked. IRP1B pulled down AGBE and the histones $\mathrm{H} 2 \mathrm{~A}$ and $\mathrm{H} 4$, but not
Cisd2. For IRP1A, all above interactions, with the exception of GlyS, were validated by PG-specific MS (Fig. 4a and Supplementary Data 1). Finally, we further validated these MS data with samples from four fly strains that expressed one of the following transgenes: apo-IRP1A (IRP1A C450S); apo-IRP1B (IRP1B $\left.{ }^{C 447 S}\right)$; non-RNA-binding IRP1A (IRP1A $3 R 3 Q)$ and IRP1B (IRP1B $\left.{ }^{3 R 3 Q}\right)$ (Supplementary Tables 1-2). This approach confirmed the results seen with the knock-in alleles, and, importantly, showed that IRP1A ${ }^{\mathrm{C} 450 \mathrm{~S}}$ failed to interact with AGBE, while Cisd2 interacted with both IRP1A variants, but none of the IRP1B proteins (Supplementary Fig. 9).

Since iron-depletion triggers the switch from holo- to apoIRP1A, one would predict that this results in cytoplasmic accumulation of IRP1A and should, therefore, alter the interactome of this protein. When we reared flies for three generations on BPS-containing media, we noticed that it takes two generations to purge IRP1A and IRP1B from PG nuclei (Supplementary Fig. 10). In the fat body, however, it takes only one generation for IRP1A to become entirely cytoplasmic, while IRP1B is still nuclear after three generations of iron-depletion (Supplementary Fig. 11), suggesting that IRPs have tissue-specific behaviours. To test whether iron-depletion affected proteinprotein interactions of IRP1A, we reared endogenously tagged IRP $1 \mathrm{~A}^{3 \mathrm{~F}}$ flies for two generations on BPS-supplemented food and conducted MS from whole-body larval samples. This strategy reduced the number of co-immunoprecipitated proteins from 166 (no BPS) to 117 (in G1 = one generation BPS) and 30 (in G2= two generations of BPS) (Supplementary Data 1). Consistent with the cytoplasmic localisation of IRP1A on BPS media, the interaction with histone $\mathrm{H} 2 \mathrm{Av}$ and $\mathrm{H} 2 \mathrm{~B}$ was lost in $\mathrm{G} 1$, and none of the four histone proteins were detected in G2, which resembles the pattern seen in the co-immunoprecipitation results with the cytoplasmic IRP1A ${ }^{\mathrm{C} 450 \mathrm{~S}}$ protein (Supplementary Data 1). Further, binding to AGBE was lost in G1 and G2, consistent with our finding that AGBE only interacts with holo-IRP1A. The interaction between IRP1A and IRP1B was lost in G2, while binding to Fer2LCH was detectable in all conditions. The topscoring protein in G2 was Cisd2/mitoNEET (Supplementary Data 1 ), indicating that the IRP1A-Cisd2/mitoNEET interaction was robust even when iron levels were low.

MitoNEET mutants phenocopy IRP1A and AGBE mutants. MitoNEET is a homodimeric Fe-S protein that resides in the 
a

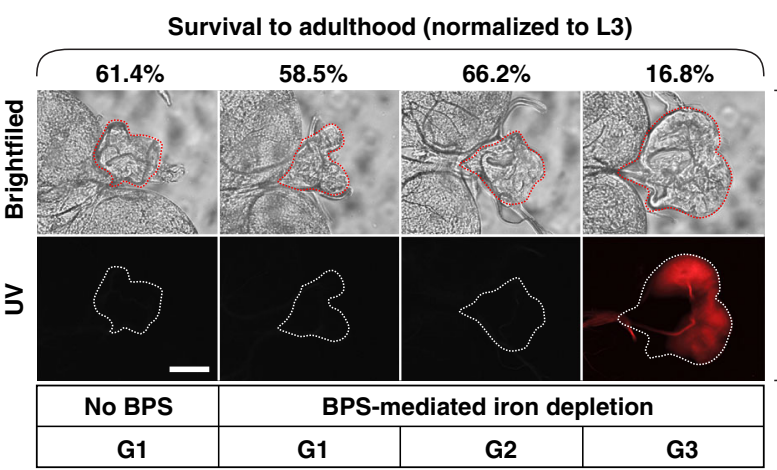

C

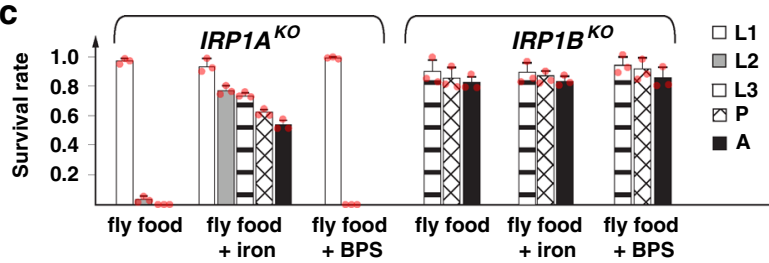

f

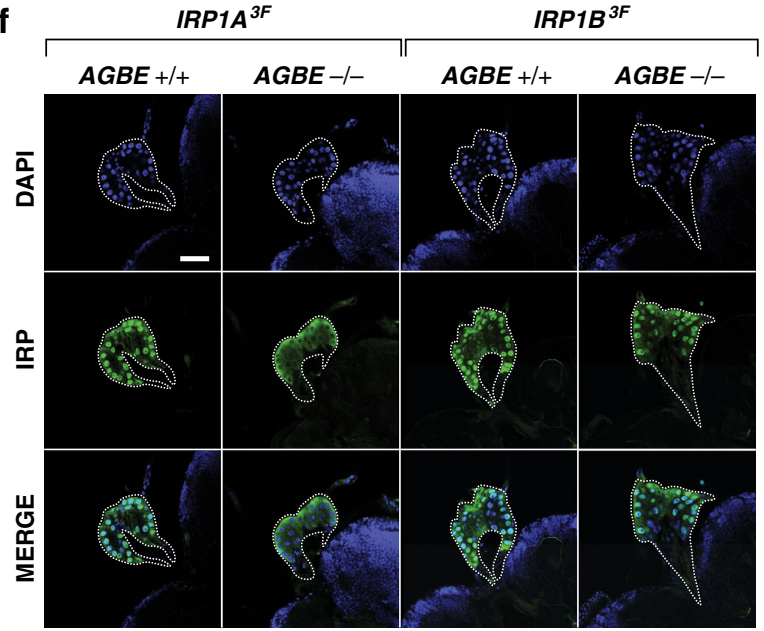

9

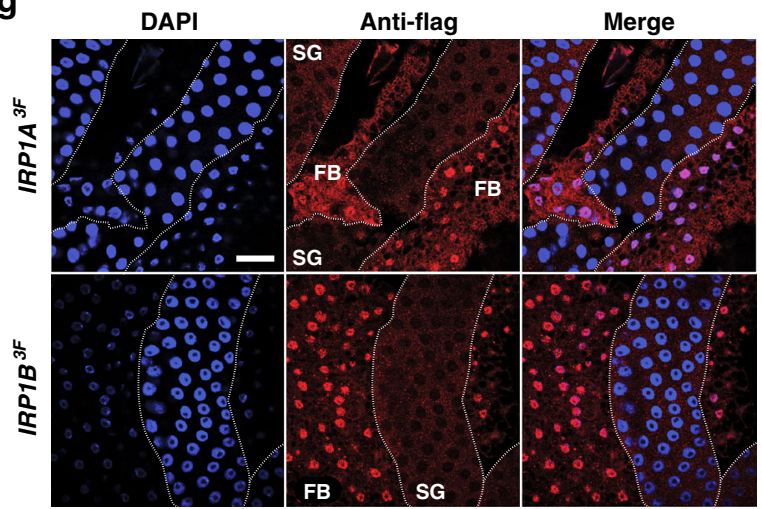

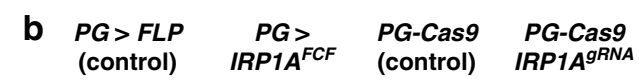
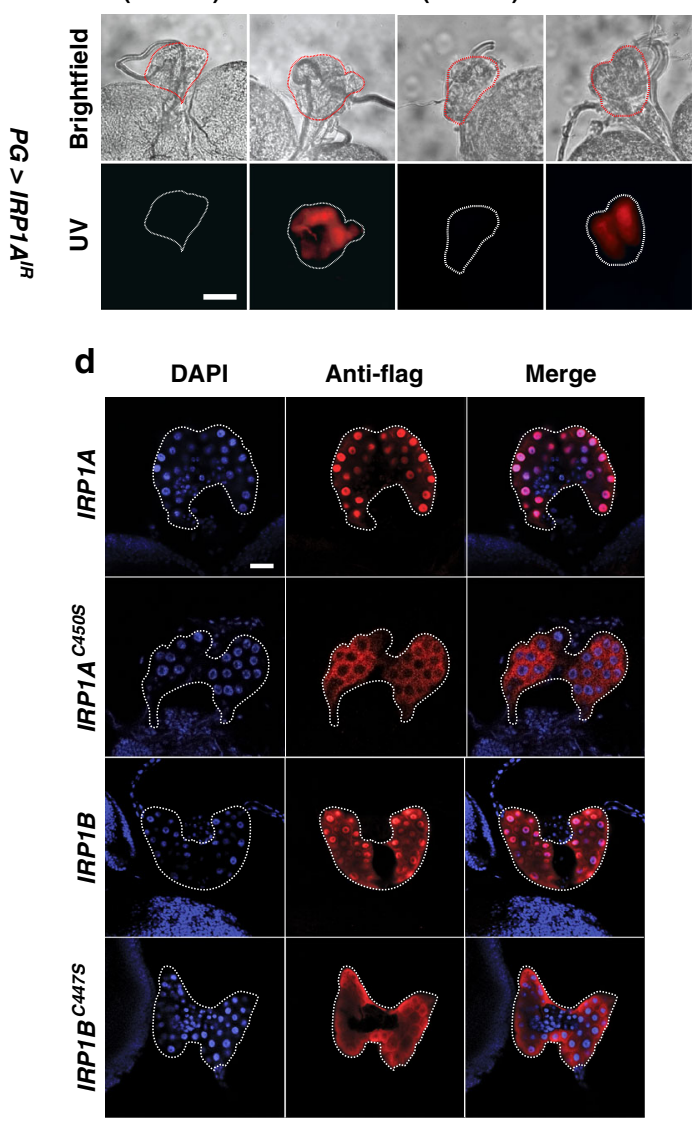

e DAPI Anti-flag Merge

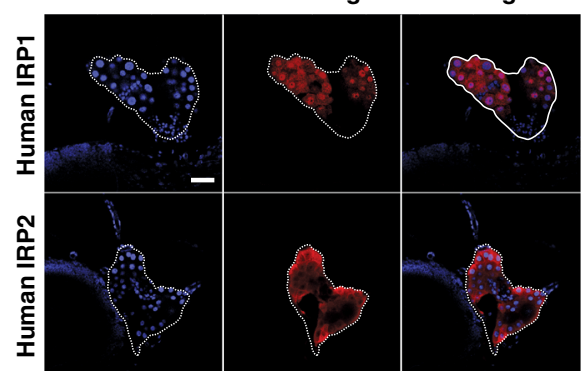

outer mitochondrial membrane, with the Fe-S cluster facing the cytosol $^{23}$. While the exact range of functions for mitoNEET remains unclear, the protein has been shown to act in the repair of oxidatively damaged vertebrate IRP1 Fe-S clusters ${ }^{23,28}$. Since the MS data suggested that Cisd2/mitoNEET represents an important link between AGBE and IRP1A, we sought validation by molecular and genetic means. First, we validated the physical interaction via co-immunoprecipitation assays in Drosophila
Schneider 2 cells (S2) cells. This showed that both AGBE and IRP1A physically interact with Cisd2 (Fig. 4c). However, this interaction was $\sim 3$-fold enhanced when AGBE and IRP1A were co-transfected together with Cisd2 (Fig. 4c, d), suggesting synergistic interactions between the three proteins.

Next, we examined Cisd 2 function by genetic means. When we depleted Cisd 2 via RNAi in the PG or examined a Cisd 2 mutant, flies survived on regular food, and displayed no protoporphyrin 
Fig. 3 IRP1 localizes to nuclei. a Protoporphyrin accumulation/autofluorescence in prothoracic glands (PG) from IRP1A-RNAi (IRP1AIR) flies reared on irondepleted (=BPS) media for three generations. Adult survival relative to last larval stage (surviving third instar larvae $=\mathrm{L} 3=100 \%$ ). Scale bar $=250 \mu \mathrm{m}$. b Protoporphyrin accumulation/autofluorescence in PGs from IRP1AFCF animals (tissue-specific excision of IRP1A, Supplementary Fig. 4) reared on iron-rich medium until L2, after which larvae were switched to BPS-supplemented food. Scale bar $=250 \mu \mathrm{m}$. c Survival of IRP1A and IRP1B null mutants (KO $=$ knockout, Supplementary Fig. 4). Error bars represent standard deviation from three biological replicates (each sample contained 50 individuals). d Subcellular localization of PG-specific, Flag-tagged IRP1A and IRP1B (PG > IRP1A/PG >IRP1B transgenic lines, Supplementary Table 1). DAPI was used to stain DNA/nuclei. Scale bar $=250 \mu \mathrm{m}$. e Subcellular localization of Flag-tagged transgenic human IRP1 and IRP2 (PG $>$ hIRP1 and PG $>$ hIRP2, Supplementary Table 1) expressed specifically in the $P G$. Scale bar $=250 \mu \mathrm{m}$. $\mathbf{f}$ Subcellular localization of Flag-tagged proteins encoded by $I R P 1 A^{3 F}$ and $I R P 1 B^{3 F}$ knock-in alleles (Supplementary Fig. 4) in control or $A G B E$ mutant backgrounds ( $A G B E^{+/+}=P G>F L P$. AGBE ${ }^{-/-}=P G>F L P ; A G B E^{F C M}$, Supplementary Fig. 4). Scale bar $=500 \mu \mathrm{m}$. $\mathbf{g}$ Subcellular localization of Flag-tagged proteins encoded by IRP1A ${ }^{3 F}$ and IRP1B $3 F$ knock-in alleles (Supplementary Fig. 4) in the fat body (FB) and salivary gland (SG). Scale bar $=500 \mu \mathrm{m}$. Source data are provided as a Source Data file.

accumulation (Fig. 4e, f). On BPS media, however, most (Cisd2 mutant) or all (Cisd2-RNAi) animals arrested development during the third instar and displayed red autofluorescence in the PG (Fig. 4e, f). We then tested whether Cisd2 and IRP1A interacted genetically, and therefore analysed RNAi lines targeting Drosophila Cisd2, IRP1A and IRP1B alone and in combination. None of the individual $P G>R N A i$ larvae displayed any overt phenotypes when reared on regular fly media. However, when we combined IRP1A- with Cisd2-RNAi, we observed strong synthetic lethality, where none of the larvae reached adulthood, and importantly, all larvae displayed protoporphyrin accumulation in the PG (Fig. 4g), indicating that both proteins participate in the same process. In contrast, the combination of $I R P 1 B$ - with Cisd2-RNAi was as ineffective as the individual lines alone. We concluded that the functional importance of the IRP1-mitoNEET interaction is conserved between vertebrates and Drosophila and that this process is essential, at least in Drosophila. Finally, we tested whether IRP1A and IRP1B could localise to nuclei in a Cisd2-mutant background. To test this, we crossed flies that harboured Flag-tagged $I R P 1 A^{3 F}$ or $I R P 1 B^{3 F}$ knock-in alleles into a Cisd2-mutant background and reared them on fly food in the presence or absence of BPS. On regular fly food, both proteins were nuclear, while exposure to BPS shifted their subcellular distribution to the cytoplasm of the PG (Fig. 4h). Control flies reared for one generation on BPS-containing food still show predominantly nuclear IRP1A and IRP1B in the PG (Supplementary Fig. 10). Taken together, these data indicate that AGBE, Cisd2/mitoNEET, and IRP1A act together to ensure that holoIRP1A remains functional and can enter the nucleus.

Nuclear IRP1. An intriguing possibility is that holo-IRP1 has additional roles in the nucleus that contribute to tissue-specific cellular iron homeostasis. This is supported by the MS data, which indicates distinct but overlapping binding behaviours by IRP1A and IRP1B to histone proteins. To examine this further, we carried out genome-wide transcript profiling of handdissected ring glands (which contain the PG) that expressed one of six Flag-tagged transgenes in a PG-specific manner: $I R P 1 A^{W T}$ and IRP1B ${ }^{W T}$, which are both wild type; IRP1A $1450 S$ and $I R P 1 B^{C 447 S}$, both of which can only assume the apo-form and are predominantly cytoplasmic; as well as $I R P 1 A^{3 R 3 Q}$ and $I R P 1 B^{3 R 3 Q}$, both of which are presumed to be non-RNA-binding and can enter nuclei (not shown) (Supplementary Table 1). The design of this approach was based on the idea that the transcriptional changes elicited by IRP $1 \mathrm{~A}^{3 \mathrm{R} 3 \mathrm{Q}}$ and IRP1B $3 \mathrm{R} 3 \mathrm{Q}$ should largely result from their nuclear function, as they are predicted to have lost RNA-binding capability. When we examined the 234 most significantly downregulated genes by IRP1A $3 R 3 \mathrm{Q}$, we noticed strong enrichment of genes involved in iron-dependent processes, most notably steroid hormone biosynthesis (Tables 1, 2 ). The results for IRP1B $3 \mathrm{R} 3 \mathrm{Q}$ were very similar (Pearson correlation $0.896, \mathrm{P}<0.001$ ), and will not be discussed separately here.
Specifically, six of the seven known Halloween enzymes were found among the 60 most strongly downregulated genes. Furthermore, other genes involved in ecdysone biosynthesis, such as transcription factors, sterol transporters, heme biosynthesis, and iron-sulfur cluster assembly proteins were also significantly enriched in this set (Table 2). Remarkably, the fold changes for these genes were highly consistent with the predicted functions of these IRP1A variants. In particular, wild type IRP1A displayed the same trend as IRP $1 \mathrm{~A}^{3 \mathrm{R} 3 \mathrm{Q}}$, but fold changes were less severe. This is consistent with the idea that wild type IRP1A is still capable of binding to mRNAs, effectively reducing nuclear IRP1A levels, resulting in similar, but reduced responses. IRP1A ${ }^{\mathrm{C} 450 \mathrm{~S}}$ is mostly cytoplasmic, but interacted weakly with histones (Supplementary Data 1), suggesting some nuclear presence. However, most gene expression changes were not significant, suggesting that IRP1 $1 \mathrm{~A}^{\mathrm{C} 450 \mathrm{~S}}$ had little influence on altering the expression profiles of this gene set. In conclusion, the use of different IRP1A variants allowed us to distinguish the different subcellular roles of IRP1A, and we could show that IRP1A 3 R3Q, but not IRP1 $\mathrm{A}^{\mathrm{C} 450 \mathrm{~S}}$, dramatically and significantly altered the expression of genes involved in iron-dependent processes.

\section{Discussion}

In this report, we demonstrated that the Drosophila glycogen branching enzyme, AGBE, has hitherto undiscovered and essential roles in the regulation of cellular iron homeostasis. We expect that AGBE's role in iron is not limited to the PG since genome-wide expression profiling indicates that AGBE is widely expressed $^{29}$. While AGBE has not been directly linked to iron homeostasis, a possible indirect link exists because mutations in RBCK1 (RanBP-type and C3HC4-type zinc finger-containing protein 1), a gene that encodes an E3 ubiquitin ligase, cause Polyglucosan Body Myopathy, a recently described glycogen storage disorder ${ }^{30}$. Intriguingly, RBCK1 was shown to control cellular iron homeostasis by degrading the oxidized form of IRP $2^{31}$, raising the idea that glycogen and iron processes are linked on multiple levels.

The finding that AGBE regulates cellular iron homeostasis led to another surprising discovery, namely that IRP1, in a tissuespecific manner, enters nuclei in its holoform to transcriptionally downregulate iron-intensive processes. Further, both AGBE and IRP1A interact with Cisd2, a close homolog of vertebrate mitoNEET, which is known to repair oxidatively damaged IRP1. We conclude that the glycogen metabolism enzyme AGBE has a "moonlighting" function in aiding Cisd 2 in this repair process, and that loss of either Cisd2- or AGBE-function results in the accumulation of damaged IRP1A, which interferes with nuclear entry (Figs. 3f, 4j, 5) and IRP1A aconitase activity (Supplementary Fig. 7C). This is consistent with our finding that only holoIRP1A can translocate to nuclei since both BPS-treatment and a mutation in a critical cysteine required for $\mathrm{Fe}-\mathrm{S}$ binding (IRP1A ${ }^{\mathrm{C} 450 \mathrm{~S}}$ and IRP1B $\mathrm{B}^{\mathrm{C} 44 \mathrm{~S}}$ ) impairs nuclear access (Fig. 3d). 
a

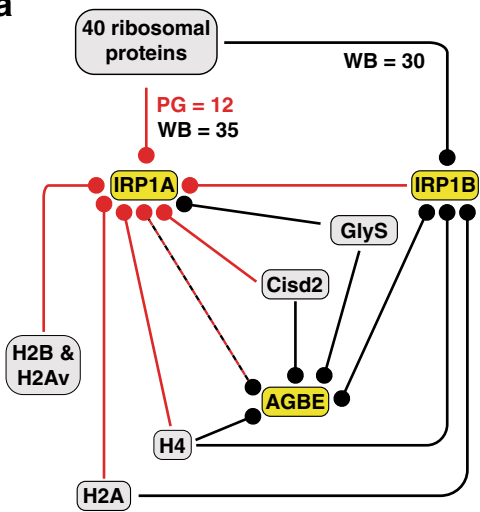

C
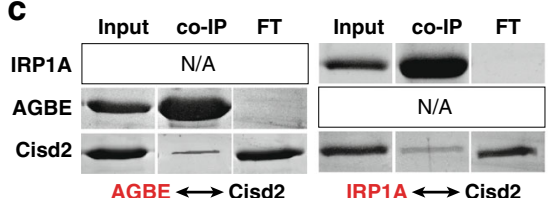

eGFP $^{\star}$

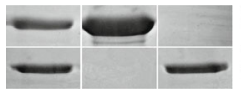

$\mathrm{eGFP}^{\mathrm{M}} \longleftrightarrow$ Cisd2

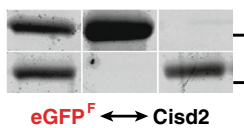

d

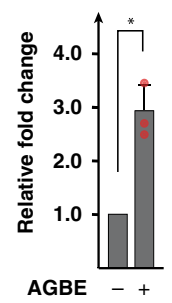

b

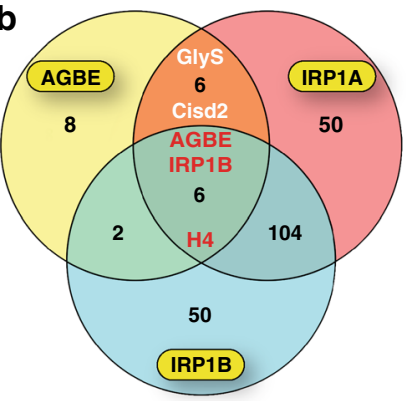

f

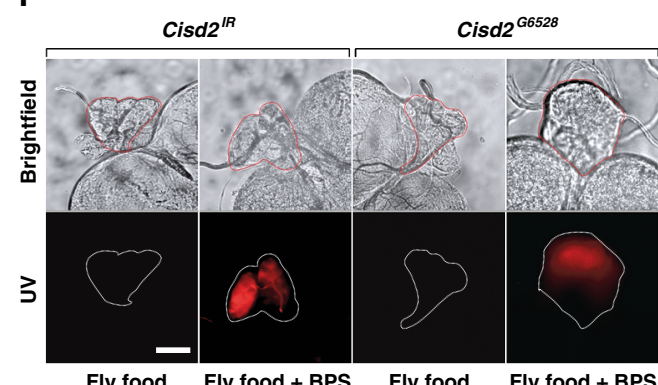

Survival to adulthood (normalized to $L 3$ )
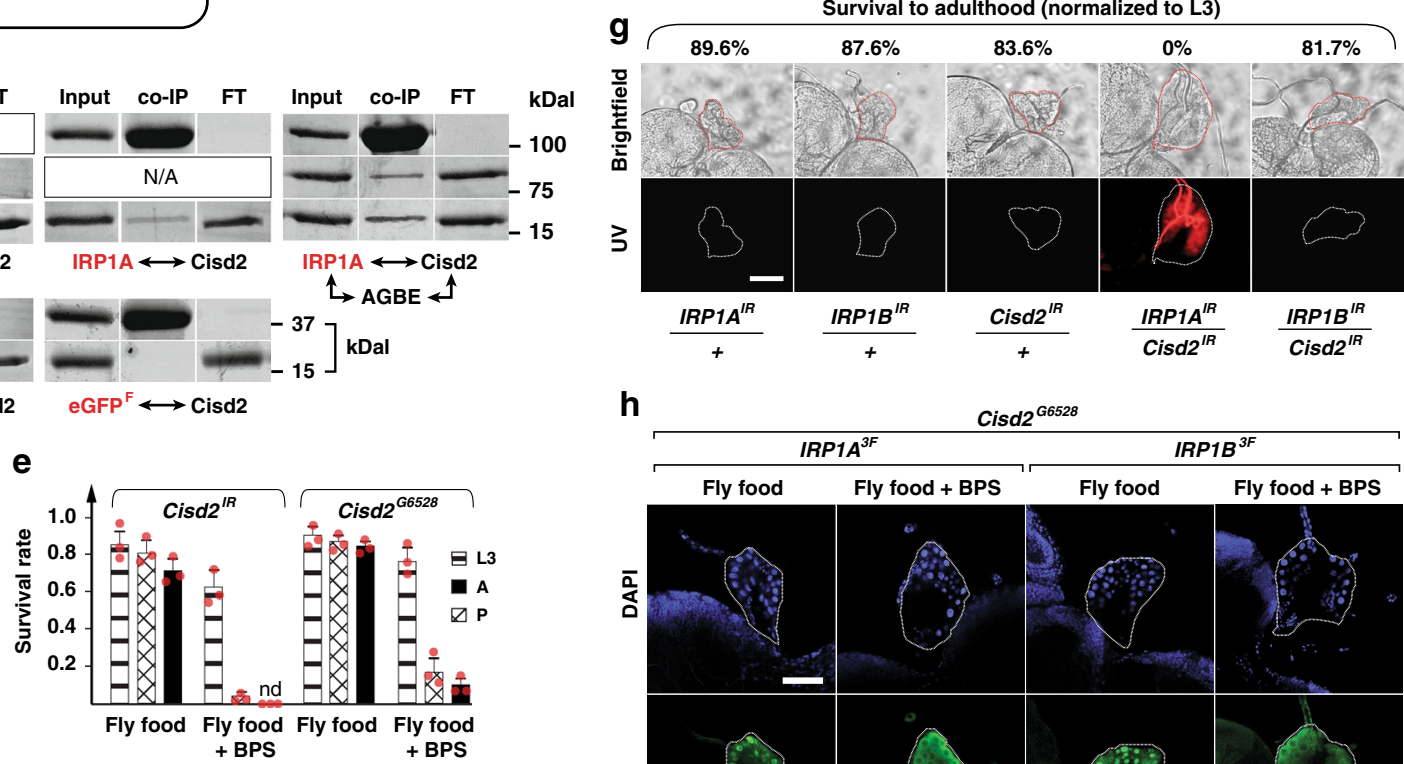

h
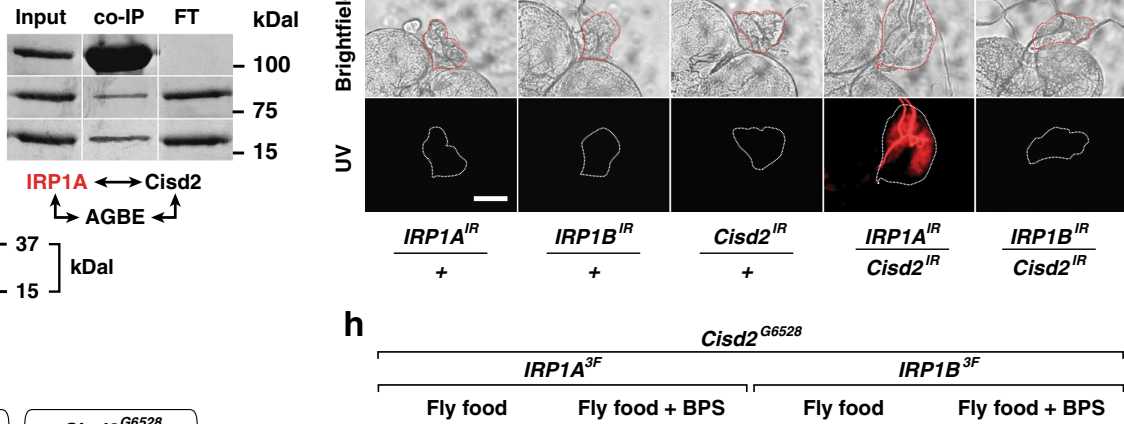

$\left.{ }_{15}^{37}\right] \mathrm{kDal}$

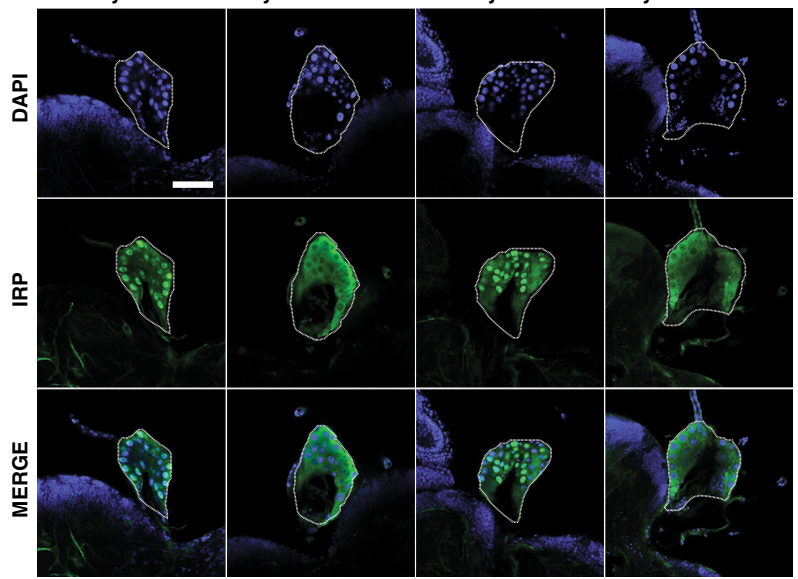

Fig. 4 Cisd2 interacts with IRP1A and AGBE. a Protein-protein interaction map. Lines carrying knock-in alleles encoding Flag-tagged AGBE, IRP1A and IRP1B (yellow boxes, Supplementary Fig. 4) were used to produce bait (circle) for immunoprecipitation followed by mass spectrometry (MS) to identify physically bound proteins to the bait. Whole-body (WB, black) and prothoracic gland samples (PG) were used. Red: detected in both PG and WB samples. Dashed line: Only WB samples were tested for AGBE. H2Av, H2A, H2B and H4 are histone proteins. GlyS = Glycogen Synthase. b Venn diagram depicting overlaps of immunoprecipitated proteins from endogenously tagged proteins (WB samples). H4 \& GlyS see A. c Co-transfection of Schneider 2 cells with plasmids encoding Myc-tagged AGBE, Flag-tagged IRP1A and HA-tagged Cisd2, followed by immunoprecipitation via anti-Myc or anti-Flag antibodies and Western blotting. Names shown in red indicate the protein used as bait. Myc-tagged and Flag-tagged enhanced GFP (eGFPM and eGFPF, respectively) served as negative controls. Presence of co-immunoprecipitated proteins were tested with anti-HA antibodies and anti-Myc antibodies. d Quantification of immunoprecipitated Cisd2 in the triple co-transfection experiment shown above in C. Graph shows relative fold change of co-immunoprecipitated Cisd2 with Flag-IRP1A as bait in the presence or absence of AGBE. Data was normalized to the amount of Cisd2 protein in the absence of co-transfected AGBE. The asterisk indicates a P-value $<0.05$ according to the Student's $t$ test. Error bars represent standard deviation based on three biological replicates. e Survival rates of Cisd2 $2^{R}$-RNAi animals and Cisd2 ${ }^{G 6528}$ mutants on fly food \pm BPS. nd $=$ not detected. Error bars represent standard deviation from three biological replicates (each sample contained 50 individuals). f Autofluorescence/protoporphyrin accumulation in prothoracic glands (PG) of $P G>C i s d 2^{R}$ and Cisd2 ${ }^{G 6528}$ larvae reared on fly food \pm BPS. Scale bar $=250 \mu \mathrm{m}$. $\mathbf{g}$ Genetic interaction between Cisd2 and IRPIA on regular (=iron-replete) fly food based on autofluorescening PGs and survival of the corresponding RNAi lines. All lines express RNAi via a PG-specific Gal4 driver (phm22-Gal4 =PG >). Scale bar $=250 \mu \mathrm{m}$. $\mathbf{h}$. Subcellular localization of Flagtagged IRP1A and IRP1B proteins expressed from knock-in alleles (Supplementary Table 1) in Cisd2 ${ }^{\mathrm{G} 6528}$ mutants reared on fly food \pm BPS. For control larvae, see Supplementary Fig. 10. Scale bar $=500 \mu \mathrm{m}$. Source data are provided as a Source Data file.

Thus, cells possess two mechanisms by which functional IRP1A can be generated. One is by "de novo" insertion via the Cytosolic Iron-sulfur protein Assembly (CIA) machinery, a highly conserved machinery that assembles and inserts [4Fe-4S] clusters into client proteins ${ }^{32}$. Once inserted, cells require a second "maintenance" process via the mitoNEET/AGBE proteins to replace oxidatively damaged clusters with functional units (Fig. 5). This elegantly explains as to why AGBE-loss-of-function 
Table 1 Transcriptional responses of expressing IRP1A alleles in the prothoracic gland.

\begin{tabular}{|c|c|c|c|c|c|c|c|c|}
\hline $\begin{array}{l}\text { Rank } \\
(n / 234)\end{array}$ & Symbol & Description & $\begin{array}{l}\text { FC } \\
\text { 3R3Q:C }\end{array}$ & $\mathbf{P}$ & $\begin{array}{l}\text { FC } \\
\text { IRP1A:C }\end{array}$ & $\mathbf{P}$ & $\begin{array}{l}\text { FC } \\
\text { C450S:C }\end{array}$ & $\mathbf{P}$ \\
\hline 6 & $d i b$ & Ecdysone biosynthesis/heme binding/P450 & -13.7 & 4.0E-03 & -2.4 & $>0.05$ & -1.7 & $>0.05$ \\
\hline 7 & phm & Ecdysone biosynthesis/heme binding/P450 & -11.7 & 1.7E-02 & -2.2 & $>0.05$ & 1.1 & $>0.05$ \\
\hline 12 & sad & Ecdysone biosynthesis/heme binding/P450 & -9.7 & $3.2 \mathrm{E}-02$ & -1.7 & 1.7E-02 & 1.2 & $>0.05$ \\
\hline 13 & Start1 & Sterol transport & -9.6 & 4.7E-03 & -3.1 & 3.3E-02 & -2.3 & $>0.05$ \\
\hline 15 & CG7322 & Short-chain dehydrogenase & -9.0 & $1.4 \mathrm{E}-02$ & -1.8 & $>0.05$ & -1.1 & $>0.05$ \\
\hline 23 & Cyp6g2 & Heme binding/P450 & -7.2 & 1.7E-02 & -1.1 & $>0.05$ & -1.2 & $>0.05$ \\
\hline 26 & spidey & Short-chain dehydrogenase & -7.0 & $1.8 \mathrm{E}-02$ & -1.6 & $2.9 \mathrm{E}-02$ & -1.6 & $1.6 \mathrm{E}-02$ \\
\hline 34 & nvd & Ecdysone biosynthesis/iron sulfur cluster & -6.1 & $1.7 \mathrm{E}-02$ & -2.1 & 8.9E-03 & -1.6 & $>0.05$ \\
\hline 35 & sro & $\begin{array}{l}\text { Ecdysone biosynthesis/short-chain } \\
\text { dehydrogenase }\end{array}$ & -6.0 & $2.1 \mathrm{E}-03$ & -4.2 & $1.3 \mathrm{E}-02$ & -1.2 & $>0.05$ \\
\hline 41 & GstE14 & $\begin{array}{l}\text { Ecdysone biosynthesis/glutathione S } \\
\text { transferase }\end{array}$ & -5.6 & $2.8 \mathrm{E}-02$ & -2.6 & $>0.05$ & -1.4 & $>0.05$ \\
\hline 44 & $N D-15$ & $\begin{array}{l}\text { NADH:ubiquinone oxidoreductase, iron- } \\
\text { sulfur subunit } 5\end{array}$ & -5.2 & $2.8 \mathrm{E}-02$ & -3.3 & $>0.05$ & -2.7 & 8.9E-03 \\
\hline 47 & ouib & Ecdysone biosynthesis/zinc finger & -4.9 & $2.4 \mathrm{E}-02$ & -2.1 & $>0.05$ & -1.8 & $>0.05$ \\
\hline 51 & spok & Ecdysone biosynthesis/heme binding/P450 & -4.8 & $3.6 \mathrm{E}-03$ & -2.4 & 2.9E-03 & -1.2 & $>0.05$ \\
\hline 52 & scu & Short-chain dehydrogenase/reductase & -4.7 & $1.1 \mathrm{E}-02$ & -1.3 & $>0.05$ & 1.2 & $>0.05$ \\
\hline 55 & Cyt-b5 & $\begin{array}{l}\text { Cytochrome b5-like heme/steroid } \\
\text { binding domain }\end{array}$ & -4.5 & $4.4 \mathrm{E}-02$ & -1.8 & $>0.05$ & -1.4 & $1.0 \mathrm{E}-02$ \\
\hline 56 & CG17928 & $\begin{array}{l}\text { Cytochrome b5-like heme/steroid } \\
\text { binding domain }\end{array}$ & -4.5 & $1.4 \mathrm{E}-02$ & -1.8 & $>0.05$ & -2.3 & 3.0E-03 \\
\hline 64 & Tig & Heme oxygenase-like & -4.2 & $6.0 \mathrm{E}-03$ & -1.7 & $>0.05$ & -1.2 & $>0.05$ \\
\hline 74 & ND-19 & NADH:ubiquinone oxidoreductase & -3.9 & 4.0E-02 & -1.5 & $>0.05$ & -1.2 & $>0.05$ \\
\hline 98 & $F d x 2$ & Ferredoxin/iron sulfur cluster assembly & -3.4 & $1.3 \mathrm{E}-03$ & -1.6 & $>0.05$ & 1.3 & $>0.05$ \\
\hline 107 & $\mathrm{Npc2a}$ & Sterol transport & -3.3 & $8.4 \mathrm{E}-03$ & -1.9 & $>0.05$ & -1.6 & $>0.05$ \\
\hline 116 & Pbgs & Heme biosynthesis & -3.2 & $6.4 \mathrm{E}-03$ & -1.5 & $>0.05$ & -1.2 & $>0.05$ \\
\hline 125 & Npcla & Sterol transport & -3.1 & $3.2 \mathrm{E}-04$ & -2.3 & 2.4E-02 & -1.2 & $>0.05$ \\
\hline 146 & Drat & Response to hypoxia & -2.9 & $1.6 \mathrm{E}-02$ & -1.4 & $>0.05$ & -1.6 & $1.1 \mathrm{E}-02$ \\
\hline 170 & CG31548 & Short-chain dehydrogenase/reductase & -2.7 & $3.1 \mathrm{E}-02$ & -1.8 & $>0.05$ & -1.4 & $>0.05$ \\
\hline 173 & CG32857 & Nfu1 homolog/iron sulfur cluster assembly & -2.7 & 3.7E-02 & -1.2 & $>0.05$ & -1.2 & $>0.05$ \\
\hline 184 & ance & Ecdysone biosynthesis/zinc finger & -2.7 & 3.7E-02 & -2.0 & $>0.05$ & -1.2 & $>0.05$ \\
\hline 202 & Alas & Heme biosynthesis & -2.6 & $1.5 \mathrm{E}-02$ & -1.0 & $>0.05$ & 12.9 & $2.6 \mathrm{E}-04$ \\
\hline 212 & CG12056 & $\begin{array}{l}\text { Cytochrome b5-like heme/steroid } \\
\text { binding domain }\end{array}$ & -2.6 & $1.7 \mathrm{E}-02$ & -1.5 & $>0.05$ & 1.1 & $>0.05$ \\
\hline 218 & CG2254 & Short-chain dehydrogenase/reductase & -2.6 & $6.7 \mathrm{E}-03$ & 1.2 & 5.1E-05 & -1.1 & $>0.05$ \\
\hline 221 & Vhl & Response to hypoxia & -2.6 & $2.0 \mathrm{E}-02$ & -1.6 & $>0.05$ & 1.1 & $>0.05$ \\
\hline
\end{tabular}

animals can only be rescued by expressing a wild type IRP1A transgene, but not by the constitutively RNA-binding form (IRP1A $\left.{ }^{\mathrm{C} 450 \mathrm{~S}}\right)$ : Sustained transgenic expression of wild type IRP1A allows cells to produce sufficient functional IRP1A before oxidative damage occurs, simply because the CIA machinery is able to maintain critical levels of holo-IRP1A, despite the absence of a functioning AGBE/mitoNEET repair machinery (Fig. 5). In contrast, IRP1A ${ }^{\mathrm{C} 450 \mathrm{~S}}$ fails to rescue, since it cannot assume the holoform that is required to enter nuclei.

What could be the biological context that requires IRP1A and IRP1B entering the nucleus? In the PG, iron demands are not only exceedingly high, but they also must change dramatically as the need for Halloween enzyme production changes during development (Supplementary Fig. 1 and Fig. 5). It is therefore plausible that once production of ecdysone has peaked, PG cells need to downregulate all processes that are tied to the synthesis of steroids. Since all but one of the ecdysone-producing Halloween enzymes require iron co-factors, it is necessary to downregulate iron-cofactor production in concert with the proteins that require them. We hypothesize that peak levels of bioavailable iron correlate with maximal nuclear activity of holo-IRP1, resulting in a downregulation of iron-dependent processes, in particular, steroid hormone biosynthesis. As such, holo-IRP1 appears to have a hitherto undescribed role in iron regulation: Apo-IRP1, as a cytoplasmic mRNA-binding protein, responds to a drop in cellular iron and facilitates an increase of bioavailable iron, yet nuclear holo-IRP1 transcriptionally downregulates iron- and heme-dependent processes once peak iron demand is over (Fig. 5).

How does IRP1 cause the coordinated transcriptional downregulation of iron-dependent processes? An attractive model is that IRP1 proteins interact with a subset of modified histone tails, rather than binding to their histone partners in a nondiscriminate fashion. We see two possible scenarios from here. First, histone-bound IRP1 could directly recruit repressive chromatin factors such as histone deacetylases or chromatin remodellers and simply act as a co-factor that serves as a readout for cellular iron concentrations. The second and perhaps more intriguing possibility is that holo-IRP1 controls nuclear citrate levels via its aconitase function to indirectly regulate histone acetylation. Nuclear citrate is converted to acetyl-CoA and oxaloacetate by nuclear ATP-citrate lyase (ACL), a metabolic enzyme with critical roles in histone acetylation ${ }^{33}$. Acetyl-CoA is the principal substrate for histone acetylation and is considered a 


\section{Table 2 Term enrichment analysis via DAVID tools.}

\begin{tabular}{|c|c|c|c|}
\hline Category & Term & $\mathbf{P}$ & $\mathbf{E}$ \\
\hline KEGG & Insect hormone biosynthesis & 9.9E-07 & 17.8 \\
\hline Keywords & Oxidoreductase & $2.9 \mathrm{E}-06$ & 2.8 \\
\hline Biological Process & Ecdysone biosynthetic process & $5.1 \mathrm{E}-05$ & 22.5 \\
\hline InterPro & NAD(P)-binding domain & $2.5 \mathrm{E}-04$ & 3.9 \\
\hline Cellular Component & Mitochondrion & 4.0E-03 & 2.1 \\
\hline InterPro & Short-chain dehydrogenase/reductase, conserved site & $9.0 \mathrm{E}-03$ & 9.1 \\
\hline Keywords & Iron & 4.0E-02 & 2.5 \\
\hline InterPro & Cytochrome b5-like heme/steroid binding domain & 4.0E-02 & 9.3 \\
\hline Molecular Function & Iron-sulfur cluster binding & 4.0E-02 & 8.9 \\
\hline Molecular Function & Heme binding & 4.6E-02 & 2.7 \\
\hline Keywords & Metalloprotease & $6.2 \mathrm{E}-02$ & 4.4 \\
\hline Biological Process & Positive regulation of ecdysteroid biosynthetic process & $9.8 \mathrm{E}-02$ & 19.5 \\
\hline
\end{tabular}

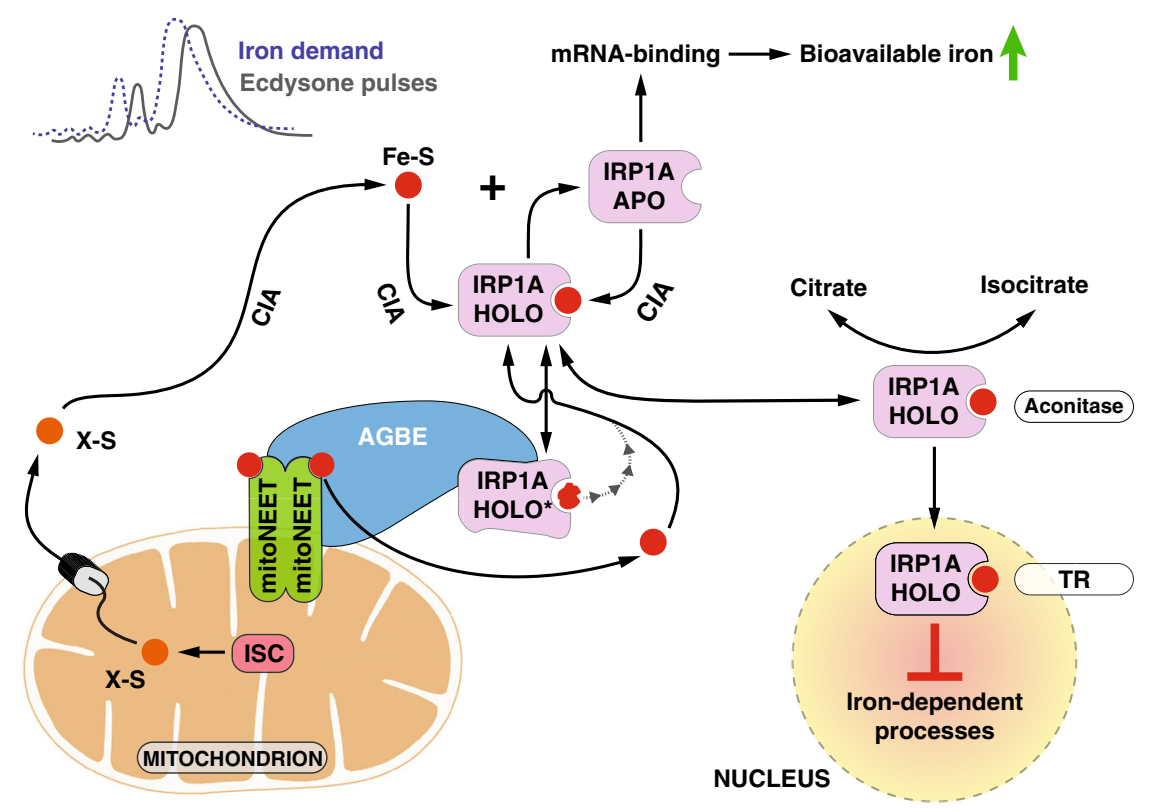

Fig. 5 Model for AGBE-mitoNEET/Cisd2-IRP1A function in tissues with dynamic iron requirements. In Drosophila, iron demand peaks prior to maximal ecdysone production to equip ecdysone-synthesizing enzymes with iron cofactors. Newly synthesized IRP1A receives Fe-S clusters (red circles) from the Cytosolic Iron-sulfur cluster Assembly (CIA) machinery, which produces [4Fe-4S] sulfur clusters from an unidentified mitochondrial precursor molecule, $\mathrm{X}-\mathrm{S}$ (produced in mitochondria by ISC = Iron-Sulfur Cluster Assembly Machinery). Oxidatively damaged IRP1A (IRP1A-HOLO*) requires the mitoNeet/ Cisd2 and AGBE proteins to replace impaired clusters with functional units. Holo-IRP1A is both needed for the aconitase function as well as nuclear entry. Loss of AGBE or mitoNEET/Cisd2 function results in a depletion of holo-IRP1A, and the concomitant loss of nuclear IRP1A, explaining why AGBE mutants cannot be rescued with the IRP1A ${ }^{C 450 S}$ form, which only assumes the apo-form since it cannot incorporate an Fe-S cluster. The model suggests that once iron demand has peaked and nuclear holo-IRP1A levels become maximal, the protein acts to throttle expression of genes acting in steroid, heme and iron metabolism in anticipation of falling iron demands. As such, IRP1A has two functions: 1. As known from mammalian cells it acts in response to low cellular iron levels as an RNA-binding protein that promotes increased iron availability, and 2. as a new function, it responds to peak iron levels as a nuclear protein to promote downregulation of processes depending on iron and heme (TR $=$ Transcriptional Regulation).

highly regulated nuclear metabolite that controls histone acetylation status ${ }^{34,35}$. Histone-bound IRP1A and IRP1B could then act by converting citrate into isocitrate and deplete Acetyl-CoA levels, thus negatively impacting gene expression by promoting histone de-acetylation.

Mutations in human GBE1 cause Andersen disease, also known as Glycogen Storage Disease Type IV (GSD IV) ${ }^{36}$, but the gene has not been linked to iron homeostasis yet. There are strong indications, however, that GBE1 has hitherto undocumented roles in vertebrate iron metabolism as well. Besides the earlier mentioned interaction with IRP1, GBE1 was identified by whole-exome sequencing as a novel mitochondrial disorder locus ${ }^{37}$, consistent with a study that found abnormal mitochondria in GSD IV patients ${ }^{38}$. Furthermore, GBE1 is transcriptionally upregulated in response to hypoxia and one of the most strongly induced genes upon nickel exposure ${ }^{39-41}$. Nickel exposure elicits hypoxic responses, and at least in vertebrates, hypoxia and iron metabolism are tightly linked ${ }^{42}$. Perhaps most intriguingly, Nrf2, a transcription factor controlling mitochondrial biogenesis and important iron metabolism genes, was shown to bind directly to 
the GBE1 promoter ${ }^{43,44}$, raising the idea that this glycogen enzyme is coordinately controlled with other key iron genes. Taken together, our findings strongly suggest that the disease etiology of GSD IV needs to be re-assessed from the perspective that GBE1 has a key role in cellular iron homeostasis, and that there must be a re-evaluation of current therapeutic strategies in the future.

The loss-of-function phenotypes for Cisd2/mitoNEET, AGBE, and IRP1A are very similar since they display protoporphyrin accumulation that disappears under iron-replete conditions. This is consistent with the idea that depleting Cisd2/mitoNEET or AGBE equates the loss of IRP1A function, as both appear to act in concert to replace damaged $\mathrm{Fe}-\mathrm{S}$ clusters in IRP1A. We have shown that IRP1A is an essential protein required for responding to low dietary iron levels, but IRP1A null mutants survive on an iron-rich diet. Therefore, our data strongly suggests that Cisd2/ AGBE are gatekeepers that ensure proper functioning of IRP1A, a function that becomes non-essential in iron-replete conditions. Vertebrates encode three mitoNEET-like proteins, Cisd1- $3^{23}$. Drosophila lacks a direct Cisd1 orthologue but harbours copies of Cisd2 and CG3420 (Cisd3). Of the two, Cisd2 is more similar to Naf- 1 and mitoNEET ${ }^{45}$. We were unable to identify any defects when disrupting CG3420 function via RNAi (not shown), suggesting that Cisd 2 is the functional equivalent of mitoNEET in Drosophila. The fact that a) fly Cisd 2 interacts physically and genetically with IRP1A and b) that mutations in either gene resulted in comparable phenotypes strongly supports the notion that IRP1A function depends on Cisd2, consistent with the finding in vertebrates that mitoNEET is involved in repairing oxidatively damaged Fe-S clusters. Similar to Drosophila IRP1A, null mutations of mouse IRP1 or IRP2 are non-lethal under normal conditions, however, the double knockout is embryonic lethal ${ }^{46-50}$. IRP1 null mutants exhibit increased blood haemoglobin levels (polycythemia) ${ }^{46,48,50}$ and one lab reported ${ }^{48}$ that these mice developed also pulmonary hypertension that was exacerbated by exposure to a low iron diet, causing premature death.

The existing parallels between vertebrate IRP1 and Drosophila IRP1A raise the interesting question as to whether vertebrate IRP1 has a nuclear role as well, and whether it is conceivable that such a function has been hitherto overlooked. Consistent with this idea, a search of a human protein-protein interaction database ${ }^{15}$ found that IRP1 interacts with Histone $2 \mathrm{Ab}^{51}$ (out of 19 reported proteins in total). In addition, we found that the presence of IRP1A in nuclei varies with tissue and nutritional conditions, raising the possibility that nuclear translocation occurs only under certain circumstances. This may be controlled by physiological parameters, depending on whether a tissue has high or normal iron requirements, and may be temporally regulated during development, as is the case for the PG. Further, we showed that the vertebrate IRP proteins use the same principles as their Drosophila counterparts for nuclear entry since only human IRP1 has the ability to translocate to Drosophila nuclei, while IRP2, which lacks an $\mathrm{Fe}-\mathrm{S}$ cluster, does not. It should also be noted that our findings were aided by the fact that the tissues we investigated are polytene, and consequently harbour, compared to most human cells, very large nuclei that allow easy visualization of nuclear proteins. Finally, we searched the literature for studies that had examined the subcellular localisation of IRP1 in more detail. To the best of our knowledge, the existing data relies solely on cell culture experiments with SW 1088 and HepG2 cells, which reported IRP1 to mainly reside in the cytosol, but also found IRP1 associated with the endoplasmic reticulum and the Golgi apparatus ${ }^{52,53}$. While the effects of hypoxic and iron-deprived conditions on IRP1 localisation were tested, iron-rich conditions were not. Taken together, we believe that IRP1, at least in certain circumstances, behaves like its Drosophila counterpart, and enters nuclei where it is physiologically relevant. Future studies will have to revisit this issue in vertebrates.

\section{Methods}

Drosophila stocks and husbandry. We obtained the following stocks from the Bloomington Drosophila Stock Center: $w^{1118}$ (\#3605), UAS-AGBE IR2 (\#42753), Cisd2 ${ }^{G 6528}$ (\#30170), Tubulin-Gal4/TM3 Ser.GFP (\#5138), UAS-eGFP (\#5431), UAS-FLP (\#4539), UAS-CD8.Venus (\#65609), Vas.Cas9 (\#51323). Stocks UAS$A G B E^{I R 1}$ (\#108087), UAS-IRP1A-RNAi (\#105583), UAS-IRP1B-RNAi (\#110637), UAS-Cisd2-RNAi (\#104501) were obtained from the Vienna Drosophila Resource Center. We used CRISPR/CAS9 to generate the following knock-in and knock-out alleles (see Supplementary Fig. 4 for details): $A G B E^{F C F}, A G B E^{F C M}, I R P 1 A^{3 F}$, $I R P 1 A^{C 450 S .3 F}, I R P 1 A^{3 R 3 Q .3 F}, I R P 1 B^{3 F}, I R P 1 A^{F C F} / T M 3$ Ser.GFP, IRP1A KO/TM3 Ser.GFP, IRP $1 B^{K O / K O}$. We also generated transgenic lines based on the PhiC31 system: UAS-3xFlag-IRP1A ${ }^{W T}, U A S-I R P 1 A^{W T}, U A S-3 x$ Flag-IRP $1 A^{C 450 S}$,

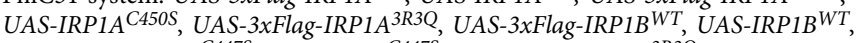
UAS-3xFlag-IRP1B ${ }^{C 447 S}, U A S-I R P 1 B^{C 447 S}, U A S-3 x$ Flag-IRP1B $^{3 R 3 Q}$, UAS-Yeast Aco $1^{W T}$, UAS-Yeast Aco ${ }^{\triangle S P}$, UAS-3xFlag-hIRP1 $1^{\text {WT }}$, UAS-3xFlag-hIRP2 ${ }^{W T}$, dU6-3IRP1AgRNA (Supplementary Table 1). $y^{1} w^{*} P$ [nos-PhiC31.NLS; $X ; P$ [carryP]attP40(II) and $y^{1} w^{*} P[$ nos-PhiC31/int.NLS]X; $P[$ carryP]attP2(III) were gifts from BestGene Inc. Phm22-Gal4 was a kind gift from Michael O'Connor's lab. Stocks were maintained on a standard cornmeal diet unless otherwise specified.

Survival studies. Regular fly food refers to "NutriFly"-based media, which follows the standard recipe from the Bloomington Drosophila Stock Center (https://bdsc. indiana.edu/information/recipes/bloomfood.html). Flies were reared at $25^{\circ} \mathrm{C}$ and $60-70 \%$ humidity. Prior to any fly-based experiments, stocks were reared on NutriFly media for at least two generations. Modified media were prepared by adding compounds (e.g., iron) during the preparation process. For iron-enriched media, a $1 \mathrm{M}$ stock solution of Ferric Ammonium Citrate (FAC) (Sigma \#F5879) was used to make NutriFly containing $1 \mathrm{mM}$ FAC. For iron-depletion media Nutrifly containing $100 \mu \mathrm{M}$ BPS (Sigma \#146617) was prepared. For egg collections, flies were allowed to lay eggs for $3 \times 1 \mathrm{~h}$ in order to reduce egg retention and minimize the presence of old embryos. For each vial, 50 embryos were then collected in 1-h intervals. Embryos were counted and transferred to vials containing appropriate media. Larval survival was scored for every stage. At least three independent crosses (=three biological replicates) were carried out per experimental condition.

Construction of transgenic lines. For transgene properties, see Supplementary Table 1. cDNAs were obtained from the Drosophila Genomic Resource Center (AGBE: \#RE12027, IRP1A: \#LD36161, IRP1B: \#LD13178, Cisd2: \#RE49709). Human IRP1 cDNA (\#HG10966-UT) and human GBE1 cDNA (\#HG18919-UT) were acquired from Sino Biological Inc, while the human IRP2 cDNA (\#OHS1770202318020) was obtained from Dharmacon. To generate equivalent expression of transgenic constructs, we used PhiC31 vectors pBID-UASC-FG (Addgene \#35201) and pBID-UASC-G (Addgene \#35202) to ensure insertion into the same locus ${ }^{54}$. Vector backbones were amplified via PCR to generate two fragments per vector and fused to cDNA fragments via the Gibson reaction. Mutations were generated via Q5 mutagenesis PCR (NEB \#M0491S) following the manufacturer's instructions. Fused fragments were cloned into DH5a E. coli competent cells, and validated by Sanger sequencing. For primers see Table 3.

Generation of CRISPR/CAS9 fly lines. We identified optimal target gRNA sites by relying on comparable results from two independent programs, "CRISPR Optimal Target Finder” (University of Wisconsin; http://tools.flycrispr.molbio. wisc.edu/targetFinder/index.php) and Harvard's "Find CRISPR" sgRNA design tool (http://www.flyrnai.org/crispr/index.html ${ }^{55}$. Target sites were confirmed by sequencing corresponding loci in the Vas.Cas9 line (Bloomington \#51323) that we used for embryo injections. CRISPR lines were generated via CRISPR/Cas9 homology-directed repair to replace endogenous alleles. Plasmids carrying gRNA target sites were cloned into pCFD3 (Addgene \#49410) for $A G B E^{F C F}, A G B E^{F C M}$, $I R P 1 A^{3 F}$ and $I R P 1 B^{3 F}$ constructs, or pCFD $5^{56,57}$ (Addgene \#73914) for $I R P 1 A^{K O}$, $I R P 1 A^{F C F}$ and $I R P 1 B^{K O}$. All donor template fragments were amplified from genomic DNA via PCR and cloned into the pDsRed-attP vector (Addgene $\# 51019)^{57}$. For primers see Table 3.

Embryo injection. PhiC31 constructs were injected at 500-600 ng/ $\mu$ l concentrations, while CRISPR plasmids were used at a concentration of $100-150 \mathrm{ng} / \mu \mathrm{l}$ for the double gRNA plasmid and 500-600 $\mathrm{ng} / \mu \mathrm{l}$ for the donor template. Injections were performed either at the University of Alberta or via GenetiVision Corporation using standard procedures. 300-500 embryos were injected per construct. Surviving adults were backcrossed to $w^{1118}$ and used to generate independent lines.

Immunostaining. Brain-ring gland complexes (BRGC) were isolated from (unless stated otherwise) $40-42 \mathrm{~h}$ 3rd instar larvae and transferred to $1 \times$ PBS. Samples were fixed in $1 \times$ PBS $4 \%$ formaldehyde (ThermoFisher \#28906) for $20 \mathrm{~min}$ at room temperature (RT) followed by washing in $1 \times$ PBS $0.3 \%$ Triton (Sigma \#T9284) 
Table 3 Primer sequences.

\section{Primer name Primer sequence $\left(5^{\prime}-3^{\prime}\right)$}

Generation of trangenic cDNA lines

attB1 IRP1A FP

attB2 IRP1A RP

attB1 IRP1B FP

attB2 IRP1B RP

attB1 yeast $A c 01$ DelSp FP

attB2 yeast $A c 01 R P$

attB1 yeast Aco1 WT FP

attB1 human IRP1 FP

attB2 human IRP1 RP

attB1 human IRP2 FP

attB2 human IRP2 RP

attB1 FG RP

miniwhite FP

miniwhite RP

attB2 FG FP

attB1 UASCG RP

attB2 UASCG FP

Yeast Aco1 WT RevMut FP

Mutagenesis of IRP1A variants

IRP1A C450S Mut FP

IRP1A C450S Mut RP

IRP1A R549Q Mut FP

IRP1A R5490 Mut RP

IRP1A R554Q Mut FP

IRP1A R554Q Mut RP

IRP1A R7120 Mut FP

IRP1A R712Q Mut RP

IRP1A R793Q Mut FP

IRP1A R7930 Mut RP

Mutagenesis of IRPIB variants

IRP1B C447S Mut FP

IRP1B C447S Mut RP

IRP1B R546Q Mut FP

IRP1B R546Q Mut RP

IRP1B R551Q Mut FP

IRP1B R5510 Mut RP

IRP1B R709Q Mut FP

IRP1B R709Q Mut RP

IRP1B R790Q Mut FP

IRP1B R790Q Mut RP

Generation of AGBE CRISPR line

AGBE LA FP

AGBE FRT LA RP

AGBE FRT Middle FP

AGBE 3xFlag RP

AGBE 3xFlag 3UTR FP

AGBE 3UTR RP

AGBE RA FRT FP

AGBE RA RP

AGBE 3xMyc RP

AGBE 3xMyc 3UTR FP

pCFD3 AGBE Left gRNA FP

pCFD3 AGBE Left gRNA RP

pCFD3 AGBE Right gRNA FP

pCFD3 AGBE Right gRNA RP

Generation of IRPIA KO CRISPR line

IRP1A LA FP

IRP1A KO LA RP

IRP1A KO RA FP

IRP1A RA RP

CAAGTTTGTACAAAAAAGCAGGCTATGTCCGGCTCCGGCGCCAATC

TCCACTTTGTACAAGAAAGCTGGGTCTAATCCAGCATTTTGCGTATC

TCAAGTTTGTACAAAAAAGCAGGCTATGTCAGGCGCCAATCCCTTC

CCCACTTTGTACAAGAAAGCTGGGTTTAAGAGAGCATTTTGCGAATCATG

CCAAGTTTGTACAAAAAAGCAGGCTGTCTCCAACTTGACTAGAGATTC

CACTTTGTACAAGAAAGCTGGGTTTTCTTCTCATCGGCCTTAATTTTATTTAAG

CCAAGTTTGTACAAAAAAGCAGGCTATGCTGTCTGCACGTTCTG

CAAGTTTGTACAAAAAAGCAGGCTATGAGCAACCCATTCGCAC

CCCACTTTGTACAAGAAAGCTGGGTCTACTTGGCCATCTTGCGGATC

TCAAGTTTGTACAAAAAAGCAGGCTATGGACGCCCCAAAAGCAG

TCCACTTTGTACAAGAAAGCTGGGTCTATGAGAATTTTCGTGCCAC

AGCCTGCTTTTTTGTACAAACTTGATACCGGTGCTTGTCATCGTC

GAGTTCGATGTGTTTATTAAGGGTATCTAGCATTAC

GTAATGCTAGATACCCTTAATAAACACATCGAACTC

ACCCAGCTTTCTTGTACAAAGTGGAGACGTAAGCTAGAGGATCTTTGTG

AGCCTGCTTTTTTGTACAAACTTGAGATATCGAGCTCTCCCGGGAATTCGGATC

ACCCAGCTTTCTTGTACAAAGTGGAGATATCGCATGCGGTACCTC

ATGCTGTCTGCACGTTCTGCCATCAAGAGACCCATTGTTCGTGGTCTTGCGACAGTCTCCAACTTGACTAGAGATTC

ATCACCTCGAGCACGAACACTTC

GGCGGCAATCACAAAGATC

CGGGCAATCAGAATTTCGAG

ACAGGACGCCACAGCAAAC

ATACTAGGGCCAATTATCTGGCCAG

TGGGATGGATCTGACCCTC

CTATTTGTCGGAACAGGGTCTAACGCCGCGCGAC

CGCGCTGCCGGTGACTTTCGTG

TGGCAGCTCACAGGATTGGGCCGCCAAG

CTGCCGTAGTCCTTGCCTAC

TCACATCCAGCACGAACACATC

TGGCCGCAATGACAACAGATC

AGTTTTGTCCGGAAACCAGAACTTCGAG

CCAGCACACACGAGGCCGTTCTTCTCGATG

CAACTATCTGGCCAGTCCTCTG

GCCCTGGTGTTGGGATGGATCTGACCCTCGAAG

TCTTGTCCGAGCAGAACATCACACCCCGTG

ATCGGGCAGCAGGCGAGGTTCTAG

CGGAAGCTCTCAGGATTGGGCCGCCAAG

CTGCCGTAGTCCTTGCCTAC

CGCTGAAGCAGGTGGAATTCTATGGCAACAGTCGGTGGCTTCTG

GAAGTTCCTATACTTTCTAGAGAATAGGAACTTCGGAAACAGCTCTGCTCCACTG

GAAGTTCCTATTCTCTAGAAAGTATAGGAACTTCGAGTGGCGACCTAATCTGTG

AATATCATGATCCTTGTAGTCTCCGTCGTGGTCCTTATAGTCCATCTAGTCACTGACGCGGGCATAAAC

ACTACAAGGATCATGATATTGATTACAAAGACGATGACGATAAGTAGCTAGTCAGACGCAATTAAC ACTACGATCGCAGGTGTGCAAAAGCAAGCCCAAATCCCTAAAATTC

ACTCATCAATGTATCTTAGAAGTTCCTATTCTCTAGAAAGTATAGGAACTTC CATTGGCCAATAACAAAG

TGCATGGAGATCTTTACTAGCATTTAGTTCTGCTCTCTTTGTTG

TGCTCGAGGTCCTCCTCGGAGATGAGCTTTTGCTCAAGATCCTCTTCAGAAATAAGTTTTTGTTCTCTAGTCACTGAC GCGGGCATAAAC

TCCGAGGAGGACCTCGAGCAGAAGTTGATCAGCGAGGAAGACTTGTAGCTAGTCAGACGCAATTAAC

GTCGGAGCAGAGCTGTTTCCGAG

AAACTCGGAAACAGCTCTGCTCC

GTCGGATTTGGGCTTGCTTTCAT

AAACATGAAAGCAAGCCCAAATC

TGTCGCCCTTCGCTGAAGCAGGTGGGTACGAGTGGGCGGGACAGAAG

GCACTACGATCGCAGGTGTGCATATAGTGGAATAATTTATCATTTTTGTGATTC

TATACGAAGTTATAGAAGAGCGCCAAACCAGTCCTGCTAAAAATGCCTAAC

GATTGACGGAAGAGCCTCGAGCGCTGGTGGTGTTGGTGATGTTGCTG

CGGCCCGGGTTCGATTCCCGGCCGATGCAGAAACATTTGTAAATTATAG GTTTTAGAGCTAGAAATAGCAAG

CFD5 IRP1A Left gRNA FP

pCFD5 IRP1A Right gRNA RP

Generation of IRPIA ${ }^{3 F}$ CRISPR line

IRP1A 3 F LA FP

IRP1A 3 F LA RP

IRP1A 3 F Middle FP

IRP1A 3 F 3xFlag RP

IRP1A 3 F 3xFlag FP

IRP1A 3 F Middle RP

pCFD3 IRP1A $3 \mathrm{~F}$ Left gRNA FP

pCFD3 IRP1A $3 F$ Left gRNA RP

pCFD3 IRP1A 3 F Right gRNA FP

pCFD3 IRP1A $3 \mathrm{~F}$ Right gRNA RP

Generation of IRP1AC450S.3F

IRP1A CRISPR Mut LA FP

IRP1A CRISPR Mut LA RP

IRP1A CRISPR Mut Middle FP

IRP1A CRISPR Mut Middle RP ATTTTAACTTGCTATTTCTAGCTCTAAAACATGGCCAAACCAGTCCTGCTTGCACCAGCCGGGAATCGAACCC

CCCTTCGCTGAAGCAGGTGGTGACCTCGGTTTCGGGGCCCAAG

GATCCGGCTGGCGAGATGTGGTCGGTGGTCACTGAATCACCGAG

CTCGGTGATTCAGTGACCACCGACCACATCTCGCCAGCCGGATC

CTTGTAATCGATGTCATGATCTTTATAATCACCGTCATGGTCTTTGTAGTCATCCAGCATTTTGCGTATCATATAG

AGATCATGACATCGATTACAAGGATGACGATGACAAGTAGTTAGTGCGTTCGTTGACTTTTATATTC

CGATCGCAGGTGTGCATAGTTAGGCATTTTTAGCAG

GTCGGCTGGCGAGATGTGGTCGG

AAACCGACCACATCTCGCCAGCC

GTCGAGCAGGACTGGTTTGGCCAT

AAACATGGCCAAACCAGTCCTGCT

CRISPR lines

TGGAATTCTTGCATGCTAGCGTGACAACTTTCATGTGCTG

TGGATATCAAGTAATAAATTTAGATAATTTTTAAG

TGGATGTGGATCATTAGATCGCTCCGAGAAGAAAATCGATATTATCCGGAAG

GCACTACGATCGCAGGTGTGCATAGTTAGGCATTTTTAGCAGGACTG

TGTATGCTATACGAAGTTATAGAAGAGCGCCTGCTGGTGAATCATCGACAAG 
Table 3 (continued)

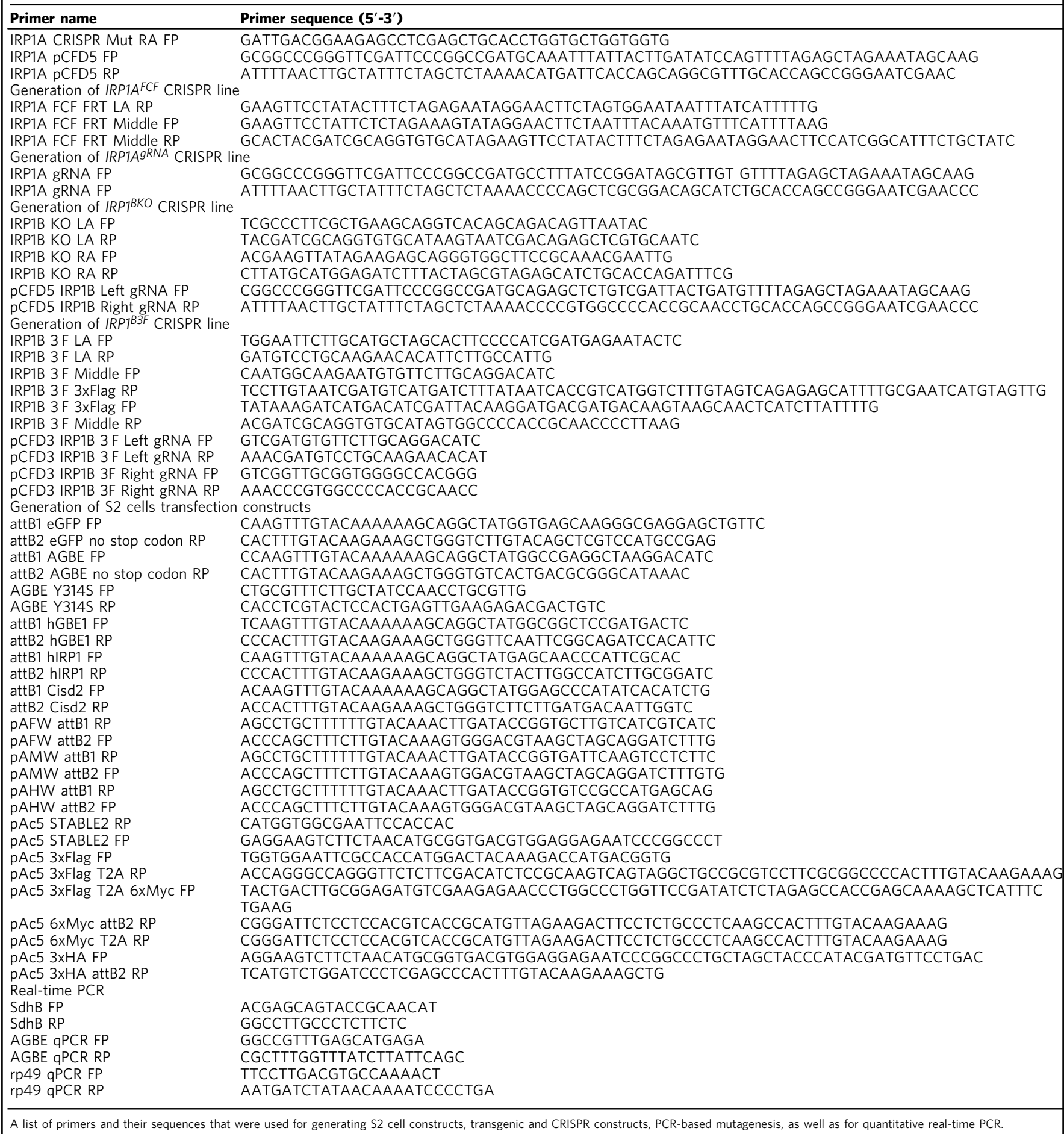

(PBS3T) for $3 \times 10 \mathrm{~min}$. Samples were blocked at RT for $1 \mathrm{~h}$ in blocking solution (1× PBS3T 5\% normal goat serum (Abcam \#ab138478)) and incubated in primary antibody dilution buffer (antibody diluted in $1 \times$ PBS3T and 1\% BSA) overnight at $4{ }^{\circ} \mathrm{C}$ with gentle shaking. Samples were then washed in $1 \times$ PBS3T for three times with $10 \mathrm{~min}$ each, incubated in secondary antibody dilution buffer for $1 \mathrm{~h}$ at RT, washed in 1× PBS3T and 1:50,000 DAPI (Cell Signaling \#4083) for three times. Samples were mounted in Vectashield mounting medium (\#VECTH1000). Pictures were taken on Nikon Eclipse 80i Confocal C2 + microscope/camera. We used the following antibodies: a monoclonal mouse anti-Flag-tag antibody (Cell Signaling \#8146S), a rabbit monoclonal anti-Flag tag antibody (Cell Signaling \#14793S), a monoclonal rabbit anti-Myc-tag antibody (Cell Signaling \#227S). Primary antibody sera were used at a ratio of 1:400 for endogenously tagged proteins and 1:1000 for transgenically expressed proteins. Secondary antibodies were obtained from Abcam and used at 1:2000 ratio, including goat anti-rabbit IgG H\&L (Alexa Fluor 488) (\#ab150077), Alexa Fluor 555 (\#ab150078), goat anti-mouse IgG H\&L Alexa Fluor 488 (\#ab150113), and Alexa Fluor 555 (\#ab150114).

Ferric iron staining. This protocol was modified from Perl's staining for iron to reduce background noise, a common issue with iron-staining techniques. 42 -h L3 larvae were washed in $1 \times$ PBS for three times and dissected for BRGC. Samples were fixed with $1 \times \mathrm{PBS} / 4 \%$ formaldehyde for $20 \mathrm{~min}$ at RT. BRGC were washed $1 \times 10 \mathrm{~min}, 1 \times 20 \mathrm{~min}$ and $1 \times 30 \mathrm{~min}$ in $1 \times \mathrm{PBS} / 0.3 \%$ Triton. Samples were incubated at RT for $1 \mathrm{~h}$ in fresh staining solution $\left(2 \% \mathrm{~K}_{4} \mathrm{Fe}(\mathrm{CN})_{6}+2 \% \mathrm{HCl}\right)$ and 
briefly washed in $1 \times \mathrm{PBS} / 0.3 \%$ Triton for $5 \times 2 \mathrm{~min}$. Samples were then incubated in $0.01 \mathrm{NaN}_{3} / 0.3 \% \mathrm{H}_{2} \mathrm{O}_{2}$ for $30 \mathrm{~min}$ at $\mathrm{RT}$ and washed $3 \times 10 \mathrm{~min}$ in $0.1 \mathrm{M}$ Phosphate buffer pH 7.0 (57.75 mM Na $2 \mathrm{HPO}_{4}$ and $\left.42.25 \mathrm{mM} \mathrm{NaH}_{2} \mathrm{PO}_{4}\right)$. Samples were then incubated for $10 \mathrm{~min}$ with fresh intensification buffer $(0.1 \mathrm{M}$ phosphate buffer $\mathrm{pH} 7.0$ containing $0.00125 \% \mathrm{DAB}$ and $0.0025 \% \mathrm{CoCl}_{2}$ ) to reduce background staining, followed by $3 \times 10 \mathrm{~min}$ wash steps in $0.1 \mathrm{M}$ phosphate buffer $\mathrm{pH}$ 7.0. Images were taken using epifluorescence camera (Nikon Digital Sight DS-U3).

RNA-sequencing. Animals were reared on standard NutriFly media (Diamed). For a single biological replicate, 50 ring glands were manually dissected in $1 \times \mathrm{PBS}$, transferred to Trizol (ThermoFisher \#15596026), and flash-frozen in liquid nitrogen for long-term storage. RNA was extracted with the RNAeasy kit (Qiagen \#74106) coupled to an on-column DNA digestion step using RNA-free DNAse (Qiagen \#79254). Extracted RNA was examined on a Bioanalyser using Agilent RNA 600 nano kit (\#5067-1511) to confirm RNA integrity. 100 ng total RNA from each sample was used for generating strand-specific RNA-Seq libraries based on the Ovation Drosophila RNA-Seq System 1-16 (Nugen \#0350-32). cDNA quality was analysed on a Bioanalyser using the high sensitivity DNA analysis kit (Agilent \#5067-4626). $100 \mathrm{ng}$ cDNA in $25 \mu \mathrm{l}$ nuclease-free water was used for RNA-Seq analysis (Genome Quebec Innovation Center at McGill University). Sequencing data was analysed using Arraystar 4.0 (DNAstar), MS Access and DAVID GO Tools $^{58}$. All RNA-Seq data has been deposited with GEO (entry \# GSE130103).

Ex vivo culturing of ring glands. In the first approach, BRGC were isolated from $w^{1118} \mathrm{~L} 3$ larvae just after the L2/L3 moult, transferred to culture medium (Schneider insect medium with $10 \%$ heat-inactivated FBS, $1 \%$ streptomycinpenicillin, $10 \mu \mathrm{g} / \mathrm{ml}$ insulin and $2 \mu \mathrm{g} / \mathrm{ml}$ ecdysone), and incubated at $25^{\circ} \mathrm{C}$. These conditions efficiently mimicked in vivo conditions and allowed physiological functions to be studied for up to $48 \mathrm{~h}^{59}$. To reduce available iron, BPS was added to the culture medium at a final concentration of $100 \mathrm{nM}$. After $24 \mathrm{~h}$, ring glands $(50$ per replicate) were transferred to Trizol for later $\mathrm{qPCR}$ analysis. In a separate approach, S2 cells were maintained under the same conditions, with a starting titer of $1 \times 10^{6}$ cells per ml. After $24 \mathrm{~h}$ at $25^{\circ} \mathrm{C}, 3 \mathrm{ml}$ were used for RNA extraction and qPCR analysis. For the in vivo approach, $w^{1118}$ larvae were initially reared on regular NutriFly food, and after the L2/L3 moult transferred to NutriFly food containing $100 \mu \mathrm{M}$ BPS. After $24 \mathrm{~h}$, ring glands were dissected and subjected to RNA extraction and qPCR analysis. For primers see Table 3.

Quantitative real-time PCR (qPCR). Extracted RNA (Qiagen RNeasy extraction kit) was reverse-transcribed via ABI High Capacity cDNA synthesis kit (ThermoFisher \#4368814). Synthesized cDNA was used for qPCR (QuantStudio 6 Flex) using KAPA SYBR Fast qPCR master mix \#Sigma KK4601). For each condition, three biological samples were each tested in triplicate. Samples were normalized to $r p 49$ based on the $\Delta \Delta C T$ method, with the exception of RNA-immunoprecipitation, where we normalized results to immunoprecipitated IRP1A protein levels. For primers see Table 3 .

Constructs for co-immunoprecipitation (S2 cells). Fragments carrying Drosophila AGBE and human GBE1 cDNAs were cloned into pAMW while Drosophila IRP1A and human IRP1 cDNAs were cloned into pAFW. The Drosophila Cisd2 cDNA was cloned into pAHW, and eGFP was cloned into pAFW as well as pAMW. This approach allowed for the generation of in-frame tagged cDNAs. We used an approach that allows for the co-expression of two cDNAs that are separated by a viral-derived $2 \mathrm{~A}$-like peptide, which is then cleaved post-translationally to yield equal amounts of both proteins ${ }^{60}$. Appropriate pairwise combinations of cDNAs encoding wild type or modified versions of 6x Myc-tagged AGBE cDNA, 6x Myc-tagged human GBE1, 3x Flag-tagged IRP1A, 3x HA-tagged Cisd2 and 3x Flagtagged eGFP (the latter served as a control) were cloned into pAc5-STABLE2-Neo (Addgene \#32426). For the triple transfection of IRP1A, AGBE and Cisd2, 3x Flagtagged IRP1A was cloned together with 6x Myc-tagged $A G B E$ as well as 3x HAtagged Cisd2 into pAc5-STABLE2-Neo, separated by viral-derived 2A-like peptides.

Transfection, co-immunoprecipitation and Western Blotting. Cells were grown in Schneider Insect medium with 10\% heat-inactivated FBS, 1\% StreptomycinPenicillin following standard procedures and transfected by the Calcium Phosphate-based method (Invitrogen). Transfected cells were lysed, and Myctagged bait proteins were immunoprecipitated using Myc-trap agarose beads (Chromotek Myc-Trap ${ }^{\circ}$-A). Flag-tagged bait proteins were immunoprecipitated using M2 Flag agarose beads (Sigma-Aldrich \#A2220) following instructions of the manufacturer. Pulled-down samples were analysed via Western Blotting. To detect 3x Myc-tagged proteins, monoclonal rabbit anti-Myc-tag antibodies (Cell Signaling \#2278S) were used at a concentration of 1:2,500 followed by incubation with a goat anti-rabbit IgG H\&L HRP secondary antibody (Abcam \#ab97051) at a ratio of 1:10,000. To detect 3x Flag-tagged proteins, monoclonal mouse anti-Flagtagged antibodies (Cell Signaling \#8146S) were used at a concentration of 1:2,500 followed by incubation with Goat anti-mouse IgG H\&L HRP secondary antibodies (Abcam \#97023) at a ratio of 1:10,000. 3x HA-tagged proteins were detected using either monoclonal mouse anti-HA-tag antibodies (Abcam \#18181) or monoclonal rabbit anti-HA-tag antibodies (Cell Signaling \#3724S) both at a ratio of 1:2,500 followed by incubation with either Goat anti-mouse or Goat antirabbit IgG H\&L HRP as a secondary antibody, respectively, at a ratio of 1:10,000 Blots were scanned for image acquisition with a ChemiDoc imaging system (BioRad). Uncropped scans of all Western Blots are provided in Supplementary Figs. 12-13.

Mass spectrometry of whole larvae. Our whole-body mass spectrometry (MS) approach was adapted from an in vivo cross-linking procedure developed for Drosophila embryos ${ }^{61,62}$. We collected 150-200 L3 larvae (40-42 h after the moult) and washed them $3 \times 5 \mathrm{~min}$ in $1 \times$ PBS. Animals were then incubated in $1 \times$ PBS with $0.1 \%$ Triton (PBS1T) $2 \times 5$ min before fixing in fresh fixative solution (1× PBS1T with $0.2 \%$ Formaldehyde) for $10 \mathrm{~min}$. The fixing solution was removed and replaced by fresh quenching solution $(0.25 \mathrm{M}$ glycine in $1 \times \mathrm{PBS} 1 \mathrm{~T})$. Animals were washed in $1 \times$ PBS1T three times before being flash-frozen in liquid nitrogen for long term storage at $-80^{\circ} \mathrm{C}$. Larvae were homogenized in $1 \mathrm{ml}$ of $1 \times$ lysis buffer (25 mM Na-HEPES pH 7.5, $75 \mathrm{mM} \mathrm{NaCl}, 0.5 \mathrm{mM}$ EDTA, $10 \%$ glycerol, $0.1 \%$ Triton X-100, proteinase inhibitor cocktail (Sigma \#11873580001)) using a Dounce homogenizer. Lysates were centrifuged at $16,000 \mathrm{~g}$ for $30 \mathrm{~min}$ at $4{ }^{\circ} \mathrm{C}$. Protein concentrations of supernatants were determined with the QubitTM Protein assay (Invitrogen \#Q33212) and served to equalize protein amounts for subsequent coimmunoprecipitation assays. The supernatants were then transferred to spin columns (Chromotek sct-50) and incubated with $40 \mu \mathrm{l}$ of anti-Flag M2 affinity gel (Sigma \#A2220) on a rotating shaker for $2 \mathrm{~h}$ at $4{ }^{\circ} \mathrm{C}$. Columns were centrifuged and treated with wash buffer 1 ( $25 \mathrm{mM}$ Na-HEPES pH 7.5, $75 \mathrm{mM} \mathrm{NaCl}, 0.5 \mathrm{mM}$ EDTA, $10 \%$ Glycerol, $0.1 \%$ Triton X-100) and wash buffer 2 (25 mM Na-HEPES $\mathrm{pH} 7.5,75 \mathrm{mM} \mathrm{NaCl}, 0.5 \mathrm{mM}$ EDTA, $10 \%$ Glycerol) for three times each. At the last step, $40 \mu \mathrm{l}$ of loading buffer $(0.125 \mathrm{M}$ Tris- $\mathrm{HCl} \mathrm{pH} 6.8,5 \%$ SDS, $0.004 \%$ Bromophenol blue, $20 \%$ glycerol, $1.43 \mathrm{M} \beta$-mercaptoethanol) was added and tubes were incubated at $95^{\circ} \mathrm{C}$ for $5 \mathrm{~min}$ before collecting samples. Samples were then loaded on a $12.5 \%$ SDS-gel, stained with Coomassie Blue and submitted for MALDI-TOF MS analysis (carried out by the Alberta Proteomics and MS Facility, University of Alberta).

In brief, we performed overnight in-gel trypsin digestion following standard procedures. Gel bands were excised and destained twice in $100 \mathrm{mM}$ ammonium bicarbonate (Sigma $\# 09830-500 \mathrm{~g}$ )/acetonitrile (Sigma \#271004) at a ratio of 50:50 $(\mathrm{v} / \mathrm{v})$. Samples were then reduced using $10 \mathrm{mM} \beta$-mercaptoethanol (Sigma \#M6250) in $100 \mathrm{mM}$ bicarbonate, followed by alkylation in $55 \mathrm{mM}$ iodoacetamide (Sigma \#I11490) in $100 \mathrm{mM}$ bicarbonate. After dehydration, a trypsin solution (Promega \#V5111) was added to cover the gel pieces at a final concentration of 6 $\mathrm{ng} / \mu \mathrm{l}$, and digested overnight $(\sim 16 \mathrm{~h})$ at RT. Tryptic peptides were first extracted from the gel using $97 \%$ water $/ 2 \%$ acetonitrile $/ 1 \%$ formic acid followed by a second extraction using $50 \%$ of the first buffer and $50 \%$ acetonitrile.

Fractions containing tryptic peptides were resolved and ionized by using nanoflow HPLC (Easy-nLC II, Thermo Scientific) coupled to an LTQ XL-Orbitrap hybrid mass spectrometer (Thermo Scientific). Nanoflow chromatography and electrospray ionization were carried out with a PicoFrit-fused silica capillary column (ProteoPepII, C18) with a $100 \mu \mathrm{m}$ inner diameter $(300 \AA$, $5 \mu \mathrm{m}$, New Objective). Peptides were loaded onto the column at a flow rate of $3000 \mathrm{nl} / \mathrm{min}$ and resolved at $500 \mathrm{nl} / \mathrm{min}$ using a $60 \mathrm{~min}$ linear gradient from 0 to $45 \% \mathrm{v} / \mathrm{v}$ aqueous acetonitrile in $0.2 \% \mathrm{v} / \mathrm{v}$ formic acid. The mass spectrometer was operated in datadependent acquisition mode, recording high-accuracy and high-resolution survey Orbitrap spectra using external mass calibration, with a resolution of 30,000 and $\mathrm{m} / \mathrm{z}$ range of $400-2000$. The fourteen most intense multiply charged ions were sequentially fragmented by using collision-induced dissociation, and the spectra of their fragments were recorded in the linear ion trap. After two fragmentations, all precursors selected for dissociation were dynamically excluded for $60 \mathrm{~s}$.

Data were processed using the Proteome Discoverer 1.4 software (Thermo Scientific), and we used the SEQUEST search algorithm (Thermo Scientific) to identify peptides in the UniProt (uniprot.org) Drosophila proteome database (ID: UP000000803). For this approach, search parameters included a precursor mass tolerance of $10 \mathrm{ppm}$ and a fragment mass tolerance of $0.8 \mathrm{Da}$. Peptides contained carbamidomethyl cysteines as static modifications and oxidized methionines and deamidated glutamines and asparagines as dynamic modifications. Minimum acceptance criteria were based on two or more peptides per detected protein, and up to two missed cleavage protease cleavage sites (C terminal sites of lysine and arginine residues) were tolerated for peptide matching. Peptide-to-spectrum matches (PSM) were set to 1 . We used the target decoy approach ${ }^{63,64}$ to estimate false discovery rates (FDRs) for peptide matches, with the strict FDR value equalling 0.01 and a relaxed FDR of 0.05 . For our analysis, the signal to noise threshold was set to 1.5. The SEQUEST protein raw score was used to calculate the final mass spectrometry score for each detected protein. The raw score represents the sum of (i) matched peptide fragment ion intensities, (ii) the number of total and matched fragment ions, and (iii) the factor that reflects the continuity of a matching ion peptide series for a given protein. SEQUEST then determines the final scores by converting the expected masses of peptide ions into a theoretical spectrum, combined with a calculation that determines the cross-correlation between the theoretical spectrum and the experimental spectrum ${ }^{65}$. In total, we analysed 21 samples representing 17 different conditions, four of which were tested 
twice (Supplementary Table 2). The reproducibility of the repeated biological samples ranged from $82.4-98.4 \%$ (Supplementary Table 2), while the overlap between equivalent IRP1A and IRP1B variants (e.g., IRP1A ${ }^{3 R 3 Q}$ vs. IRP1B ${ }^{3 R 3 Q}$ ) ranged from $63.8 \%$ to $66.3 \%$. In total, five control samples were used (Supplementary Table 2). Proteins detected in any of the control samples were removed from experimental results.

Mass spectrometry of the prothoracic gland (PG). For PG-specific MS we separated hand-dissected BRGC into individual cells. We used larvae that expressed Venus-tagged CD8 (UAS-CD8.Venus, Bloomington stock \#65609) in a PG-specific manner. CD8 localises to the cell membrane, allowing purification of PG cells from unlabelled cells ${ }^{66}$. BRGC were collected in ex vivo medium (Schneider insect medium with 10\% heat-inactivated FBS, $1 \%$ streptomycinpenicillin, $10 \mu \mathrm{g} / \mathrm{ml}$ insulin and $2 \mu \mathrm{g} / \mathrm{ml}$ ecdysone) containing a proteinase inhibitor cocktail (Sigma \#11873580001). Dissection times were limited to $1 \mathrm{~h}$ to minimize physiological changes. Samples were incubated in $1 \times$ PBS1T for $2 \times 5$ min before being fixed in fresh fixing solution ( $1 \times$ PBS1T containing $0.2 \%$ formaldehyde) for 10 min. Fixing solution was removed and replaced by fresh quenching solution $(0.25 \mathrm{M}$ glycine in $1 \times$ PBS1T). Samples were washed three times in $1 \times$ PBS1T, followed by immersion in $1 \times \mathrm{PBS1T} / 25 \%$ glycerol and flash-frozen in liquid nitrogen for long-term storage at $-80^{\circ} \mathrm{C}$. For knock-in derived proteins we collected $1.0 \mathrm{ml}$ containing the equivalent of $\sim 15,000$ BRGCs isolated from $40-42 \mathrm{~h}$ old L3, while roughly half the amount was used for flies with transgenically produced protein. Samples were removed from $-80^{\circ} \mathrm{C}$ and thawed gradually for 15 min at $-20^{\circ} \mathrm{C}$ followed by $15 \mathrm{~min}$ at $4{ }^{\circ} \mathrm{C}$ until completely thawed. Tissue samples were then incubated in cell dissociation buffer (CMF buffer with $1 \mathrm{mg} / \mathrm{ml}$ collagenase, $1 \mathrm{mg} / \mathrm{ml}$ papain) for $30 \mathrm{~min}$ at $30^{\circ} \mathrm{C}$. The digestion was terminated by adding $4 \mathrm{x}$ volumes of CMF to the dissociation reaction. Samples were left at RT for $5 \mathrm{~min}$ before being centrifuged at $1000 \mathrm{~g}$ for $1 \mathrm{~min}$. Cells were $3 \times$ washed in PBS1T and incubated with IgG beads that had been cross-linked with mouse CD8 antibody (\#ab82005) for $30 \mathrm{~min}$, followed by three brief washes in PBS1T, and an elution step (0.1 M citrate $\mathrm{pH} 2.3)$ to release PG cells from beads. All subsequent steps for protein extraction and immunoprecipitation were as described for wholebody MS. All MS proteomics data have been deposited to the ProteomeXchange Consortium ${ }^{67}$ via the PRIDE $^{68}$ partner repository with identifier \#PXD013499.

Quantitative RNA-immunoprecipitation (RIP). Our in vivo RIP approach was adapted from different cell culture protocols $s^{69-71}$. As controls, we used $w^{1118}$, which is the parental strain for our transgenic and mutant lines and thus harbours no tagged genes. To immunoprecipitate IRP1A, we used 3x Flag-tagged CRISPR/ Cas9-generated knock-in alleles, namely IRP1A ${ }^{3 F}, I R P 1 A^{C 450 S .3 F}$, and IRP1A ${ }^{3 R 3 Q .3 F}$, representing tagged wild type, constitutively RNA-binding and nonRNA-binding forms of IRP1A, respectively. We collected $200 \mathrm{~L} 3$ larvae (staged at $40 \mathrm{~h}$ after the L2/L3 moult) per sample. Larvae were washed for $3 \times 5 \mathrm{~min}$ in PBS, flash-frozen in liquid nitrogen and stored at $-80^{\circ} \mathrm{C}$. Larvae were homogenized in $1 \mathrm{ml}$ lysis buffer $(150 \mathrm{mM} \mathrm{KCl}, 25 \mathrm{mM}$ Tris- $\mathrm{HCl} \mathrm{pH}$ 7.4, $5 \mathrm{mM}$ EDTA, 0.5\% v/v Nonidet P-40, $1 \times$ proteinase inhibitor cocktail, $100 \mathrm{U} / \mathrm{ml}$ RNase inhibitor (NEB \#M0314S) using a Dounce homogenizer. Lysates were centrifuged at $12,000 \mathrm{~g}$ for $30 \mathrm{~min}$ at $4^{\circ} \mathrm{C}$. Supernatants were transferred and filtered through a $0.45 \mu \mathrm{m}$ syringe filter (Sigma \#CLS431225-50EA). Flow-through samples were incubated with $300 \mu$ l equilibrated anti-Flag M2 affinity gel solution on a rotating platform for $4 \mathrm{~h}$ at $4{ }^{\circ} \mathrm{C}$ followed by centrifugation at $12,000 \mathrm{~g}$ for $30 \mathrm{~min}$ at $4{ }^{\circ} \mathrm{C}$. The supernatant was removed, and the affinity gel washed in 10x volume of lysis buffer for 2 $\times 5 \mathrm{~min}$. $5 \%$ of the final volume was saved for Western Blotting to determine IRP1A levels to adjust sample input for RIP (Fig. 2c). Western Blots were scanned with the ChemiDoc imaging system (Bio-Rad), and bands were quantified using ImageJ following standard procedures. The remaining $95 \%$ was used for Trizolbased RNA extraction followed by qPCR for $S d h B$, which harbours a validated IRE ${ }^{72,73}$. For primers see Table 3 .

Measuring IRP1A and IRP1B aconitase activity (S2 cells). S2 cells were grown in Schneider Insect medium with 10\% heat-inactivated FBS/1\% Streptomycin-Penicillin and transfected by the Calcium Phosphate-based method (Invitrogen). Transfected cells were lysed, and IRP1 protein levels were evaluated as follows: From each sample, $50 \%$ of the lysate was used to immunoprecipitate IRP1A or IRP1B, and proteins were separated via SDS-PAGE. This was followed by Coomassie Blue staining of the gel to evaluate IRP1A and IRP1B protein levels, and cell lysate amounts used for aconitase assays were normalized accordingly. Aconitase activity was determined by measuring the rate of NADPH production via absorbance at $340 \mathrm{~nm}$ every $5 \mathrm{~min}$ (Aconitase$340^{\mathrm{TM}}$ kit, OxisResearch 21041, DU-730 UV/Vis Spectrophotometer). The absorbance rate was normalized relative to the rate of untransfected S2 cells, which served as a negative control for background aconitase activity.

Measuring IRP1A and IRP1B aconitase activity in vivo. We measured aconitase activity from both transgenically produced IRP1 (Supplementary Fig. 7) as well as from knock-in alleles (Fig. 2d). For the former, we collected 200-250 L1 larvae that ubiquitously expressed transgenic IRP1A or IRP1B alleles (tub-Gal4 $>U A S$ CDNA) and washed them $3 \times 5 \mathrm{~min}$ in $1 \times$ PBS. To measure the IRP1 aconitase activity in an $A G B E$ mutant background, we generated lines carrying either transgenic $t u b>I R P 1 A$ or $t u b>I R P 1 B$ together with transgenic UAS-FLP-cDNA and the $A G B E^{F C F}$ knock-in allele to remove $A G B E$ ubiquitously. For corresponding controls, we used the same combination, except that we replaced UAS$F L P$-cDNA with a $U A S>e G F P$-cDNA transgene. To evaluate the aconitase activity produced by knocked-in IRP1A alleles, we collected $200 \mathrm{~L} 3$ larvae (staged at $42 \mathrm{~h}$ after the L2/L3 moult) carrying different IRP1A alleles in an IRP1B null mutant background to eliminate IRP1B aconitase activity. Larvae were homogenized in $1 \mathrm{ml}$ of $1 \times$ Lysis buffer ( $25 \mathrm{mM} \mathrm{Na}$-HEPES pH 7.5, $75 \mathrm{mM} \mathrm{NaCl}, 0.5$ mM EDTA, $10 \%$ glycerol, $0.1 \%$ Triton X-100, proteinase inhibitor cocktail). To reduce the contribution of mitochondrial aconitase (Acon), we removed the mitochondrial fraction via ultracentrifugation at $20,000 \mathrm{~g}$ and $4{ }^{\circ} \mathrm{C}$. We normalized samples based on immunoprecipitated tagged protein levels (i.e., IRP1A variants), which we evaluated via Western Blotting (Fig. 2d, not shown in Supplementary Fig. 7), as described in the quantitative RIP section above. Aconitase activity was determined as described for S2 cells. For the knocked-in CRISPR alleles of $I R P 1 A$, we used IRP1B null mutants as controls, which harbour a wild type copy of IRPIA.

Reporting summary. Further information on research design is available in the Nature Research Reporting Summary linked to this article.

\section{Data availability}

The source data underlying Figs. 1g-h, 2a-d, 3a, 3c. 4e-g, 4i and Supplementary Fig. 7 are provided as a Source Data file at FigShare (https://figshare.com/s/ eb4451ceeae5e0fd926f) with DOI information https://doi.org/10.6084/m9. figshare.8001809. Mass spectrometry proteomics data relating to Fig. 4a, b and Supplemental Tables 3-6 have been deposited with the ProteomeXchange Consortium via the PRIDE partner repository (identifier \#PXD013499). RNA-Seq data have been deposited with GEO (https://www.ncbi.nlm.nih.gov/geo/query/acc.cgi?acc $=$ GSE130103).

Received: 22 June 2018; Accepted: 22 October 2019; Published online: 29 November 2019

\section{References}

1. Lill, R. Function and biogenesis of iron-sulphur proteins. Nature 460, 831-838 (2009).

2. Muckenthaler, M. U., Rivella, S., Hentze, M. W. \& Galy, B. A red carpet for iron metabolism. Cell 168, 344-361 (2017).

3. Anderson, C. P., Shen, M., Eisenstein, R. S. \& Leibold, E. A. Mammalian iron metabolism and its control by iron regulatory proteins. Biochim. Biophys. Acta 1823, 1468-1483 (2012).

4. Walden, W. E. et al. Structure of dual function iron regulatory protein 1 complexed with ferritin IRE-RNA. Science 314, 1903-1908 (2006).

5. Volz, K. The functional duality of iron regulatory protein 1. Curr. Opin. Struct. Biol. 18, 106-111 (2008).

6. Papanikolaou, G. \& Pantopoulos, K. Systemic iron homeostasis and erythropoiesis. IUBMB Life 69, 399-413 (2017).

7. Danielsen, E. T. et al. A Drosophila genome-wide screen identifies regulators of steroid hormone production and developmental timing. Dev. Cell 37, 558-570 (2016).

8. Ou, Q. et al. The insect prothoracic gland as a model for steroid hormone biosynthesis and regulation. Cell Rep. 16, 247-262 (2016).

9. Yoshiyama-Yanagawa, T. et al. The conserved rieske oxygenase DAF-36/ Neverland Is a novel cholesterol-metabolizing enzyme. J. Biol. Chem. 286, 25756-25762 (2011).

10. Heinemann, I. U., Jahn, M. \& Jahn, D. The biochemistry of heme biosynthesis. Arch. Biochem. Biophys. 474, 238-251 (2008).

11. Nagababu, E. \& Rifkind, J. M. Formation of fluorescent heme degradation products during the oxidation of hemoglobin by hydrogen peroxide. Biochem. Biophys. Res. Commun. 247, 592-596 (1998).

12. Caceres, L. et al. Nitric oxide coordinates metabolism, growth, and development via the nuclear receptor E75. Genes Dev. 25, 1476-1485 (2011).

13. Cappellini, M. D., Brancaleoni, V., Graziadei, G., Tavazzi, D. \& Di Pierro, E. Porphyrias at a glance: diagnosis and treatment. Intern. Emerg. Med. 5(Suppl 1), S73-S80 (2010).

14. Orhan Akman, H. et al. A novel mouse model that recapitulates adult-onset glycogenosis type 4. Hum. Mol. Genet 24, 6801-6810 (2015).

15. Stark, C. et al. BioGRID: a general repository for interaction datasets. Nucleic Acids Res. 34, D535-D539 (2006).

16. Wan, C. et al. Panorama of ancient metazoan macromolecular complexes. Nature 525, 339-344 (2015).

17. Lind, M. I. et al. Of two cytosolic aconitases expressed in Drosophila, only one functions as an iron-regulatory protein. J. Biol. Chem. 281, 18707-18714 (2006). 
18. Philpott, C. C., Klausner, R. D. \& Rouault, T. A. The bifunctional ironresponsive element binding protein/cytosolic aconitase: the role of active-site residues in ligand binding and regulation. Proc. Natl Acad. Sci. USA 91, 7321-7325 (1994).

19. Regev-Rudzki, N., Karniely, S., Ben-Haim, N. N. \& Pines, O. Yeast aconitase in two locations and two metabolic pathways: seeing small amounts is believing. Mol. Biol. Cell 16, 4163-4171 (2005).

20. Huynh, N., Zeng, J., Liu, W. \& King-Jones, K. A. Drosophila CRISPR/Cas9 toolkit for conditionally manipulating gene expression in the prothoracic gland as a test case for polytene tissues. G3 (Bethesda) 8, 3593-3605 (2018).

21. Paraskeva, E., Gray, N. K., Schläger, B., Wehr, K. \& Hentze, M. W. Ribosomal pausing and scanning arrest as mechanisms of translational regulation from cap-distal iron-responsive elements. Mol. Cell Biol. 19, 807-816 (1999).

22. Muckenthaler, M., Gray, N. K. \& Hentze, M. W. IRP-1 binding to ferritin mRNA prevents the recruitment of the small ribosomal subunit by the capbinding complex eIF4F. Mol. Cell 2, 383-388 (1998).

23. Mittler, R. et al. NEET proteins: a new link between iron metabolism, reactive oxygen species, and cancer. Antioxid Redox Signal 30, 1083-1095 (2018).

24. Lipper, C. H. et al. Cancer-related NEET proteins transfer $2 \mathrm{Fe}-2 \mathrm{~S}$ clusters to anamorsin, a protein required for cytosolic iron-sulfur cluster biogenesis. PLoS One 10, e0139699 (2015).

25. Tan, G. et al. His-87 ligand in mitoNEET is crucial for the transfer of iron sulfur clusters from mitochondria to cytosolic aconitase. Biochem. Biophys. Res. Commun. 470, 226-232 (2016).

26. Zuris, J. A. et al. Facile transfer of [2Fe-2S] clusters from the diabetes drug target mitoNEET to an apo-acceptor protein. Proc. Natl Acad. Sci. USA 108, 13047-13052 (2011)

27. Inupakutika, M. A. et al. Phylogenetic analysis of eukaryotic NEET proteins uncovers a link between a key gene duplication event and the evolution of vertebrates. Sci. Rep. 7, 42571 (2017).

28. Ferecatu, I. et al. The diabetes drug target MitoNEET governs a novel trafficking pathway to rebuild an Fe-S cluster into cytosolic aconitase/iron regulatory protein 1. J. Biol. Chem. 289, 28070-28086 (2014).

29. Chintapalli, V. R., Wang, J. \& Dow, J. A. Using FlyAtlas to identify better Drosophila melanogaster models of human disease. Nat. Genet. 39, 715-720 (2007).

30. Nilsson, J. et al. Polyglucosan body myopathy caused by defective ubiquitin ligase RBCK1. Ann. Neurol. 74, 914-919 (2013).

31. Yamanaka, K. et al. Identification of the ubiquitin-protein ligase that recognizes oxidized IRP2. Nat. Cell Biol. 5, 336-340 (2003).

32. Netz, D. J., Mascarenhas, J., Stehling, O., Pierik, A. J. \& Lill, R. Maturation of cytosolic and nuclear iron-sulfur proteins. Trends Cell Biol. 24, 303-312 (2014).

33. Wellen, K. E. et al. ATP-citrate lyase links cellular metabolism to histone acetylation. Science 324, 1076-1080 (2009).

34. Moussaieff, A. et al. Glycolysis-mediated changes in acetyl-CoA and histone acetylation control the early differentiation of embryonic stem cells. Cell Metab. 21, 392-402 (2015).

35. Sutendra, G. et al. A nuclear pyruvate dehydrogenase complex is important for the generation of acetyl-CoA and histone acetylation. Cell 158, 84-97 (2014).

36. Li, S. C. et al. Glycogen storage disease type IV: novel mutations and molecular characterization of a heterogeneous disorder. J. Inherit. Metab. Dis. 33(Suppl 3), S83-S90 (2010).

37. Pronicka, E. et al. New perspective in diagnostics of mitochondrial disorders: two years' experience with whole-exome sequencing at a national paediatric centre. J. Transl. Med. 14, 174 (2016).

38. Nolte, K. W., Janecke, A. R., Vorgerd, M., Weis, J. \& Schröder, J. M. Congenital type IV glycogenosis: the spectrum of pleomorphic polyglucosan bodies in muscle, nerve, and spinal cord with two novel mutations in the GBE1 gene. Acta Neuropathol. 116, 491-506 (2008).

39. Zhao, J. et al. Nickel-induced 1,4-alpha-glucan branching enzyme 1 upregulation via the hypoxic signaling pathway. Toxicol. Appl Pharm. 196, 404-409 (2004).

40. Pescador, N. et al. Hypoxia promotes glycogen accumulation through hypoxia inducible factor (HIF)-mediated induction of glycogen synthase 1. PLoS One 5, e9644 (2010).

41. Buart, S. et al. Transcriptional response to hypoxic stress in melanoma and prognostic potential of GBE1 and BNIP3. Oncotarget 8, 108786-108801 (2017).

42. Mandilaras, K., Pathmanathan, T. \& Missirlis, F. Iron absorption in Drosophila melanogaster. Nutrients 5, 1622-1647 (2013).

43. Uruno, A. et al. Nrf2-mediated regulation of skeletal muscle glycogen metabolism. Mol. Cell Biol. 36, 1655-1672 (2016).

44. Kerins, M. J. \& Ooi, A. The roles of NRF2 in modulating cellular iron homeostasis. Antioxid Redox Signal 29, 1756-1773 (2017).
45. Hu, Y. et al. An integrative approach to ortholog prediction for diseasefocused and other functional studies. BMC Bioinform. 12, 357 (2011).

46. Anderson, S. A. et al. The IRP1-HIF- $2 \alpha$ axis coordinates iron and oxygen sensing with erythropoiesis and iron absorption. Cell Metab. 17, 282-290 (2013).

47. Galy, B., Ferring, D., Benesova, M., Benes, V. \& Hentze, M. W. Targeted mutagenesis of the murine IRP1 and IRP2 genes reveals context-dependent RNA processing differences in vivo. RNA 10, 1019-1025 (2004).

48. Ghosh, M. C. et al. Deletion of iron regulatory protein 1 causes polycythemia and pulmonary hypertension in mice through translational derepression of HIF2a. Cell Metab. 17, 271-281 (2013).

49. LaVaute, T. et al. Targeted deletion of the gene encoding iron regulatory protein-2 causes misregulation of iron metabolism and neurodegenerative disease in mice. Nat. Genet 27, 209-214 (2001)

50. Wilkinson, N. \& Pantopoulos, K. IRP1 regulates erythropoiesis and systemic iron homeostasis by controlling HIF2a mRNA translation. Blood 122, 1658-1668 (2013).

51. Kristensen, A. R., Gsponer, J. \& Foster, L. J. A high-throughput approach for measuring temporal changes in the interactome. Nat. Methods 9, 907-909 (2012).

52. Christova, T. \& Templeton, D. M. Effect of hypoxia on the binding and subcellular distribution of iron regulatory proteins. Mol. Cell Biochem. 301, 21-32 (2007)

53. Patton, S. M., Piñero, D. J., Surguladze, N., Beard, J. \& Connor, J. R. Subcellular localization of iron regulatory proteins to Golgi and ER membranes. J. Cell Sci. 118, 4365-4373 (2005).

54. Wang, J. W., Beck, E. S. \& McCabe, B. D. A modular toolset for recombination transgenesis and neurogenetic analysis of Drosophila. PLoS One 7, e42102 (2012).

55. Gratz, S. J. et al. Highly specific and efficient CRISPR/Cas9catalyzed homology-directed repair in Drosophila. Genetics 196, 961-971 (2014).

56. Port, F., Chen, H. M., Lee, T. \& Bullock, S. L. Optimized CRISPR/Cas tools for efficient germline and somatic genome engineering in Drosophila. Proc. Natl Acad. Sci. USA 111, E2967-E2976 (2014).

57. Port, F. \& Bullock, S. L. Augmenting CRISPR applications in Drosophila with tRNA-flanked sgRNAs. Nat. Methods 13, 852-854 (2016).

58. Huang, D. W. et al. The DAVID Gene Functional Classification Tool: a novel biological module-centric algorithm to functionally analyze large gene lists. Genome Biol. 8, R183 (2007).

59. Prithviraj, R., Trunova, S. \& Giniger, E. Ex vivo culturing of whole, developing Drosophila brains. J. Vis. Exp. 65, 4270 (2012).

60. González, M. et al. Generation of stable Drosophila cell lines using multicistronic vectors. Sci. Rep. 1, 75 (2011).

61. Gao, M., McCluskey, P., Loganathan, S. N. \& Arkov, A. L. An in vivo crosslinking approach to isolate protein complexes from Drosophila embryos. J. Vis. Exp. doi 10.3791/51387 (2014).

62. Gerace, E. \& Moazed, D. Affinity pull-down of proteins using Anti-FLAG M2 agarose beads. Methods Enzymol. 559, 99-110 (2015).

63. Elias, J. E. \& Gygi, S. P. Target-decoy search strategy for increased confidence in large-scale protein identifications by mass spectrometry. Nat. Methods $\mathbf{4}$, 207-214 (2007)

64. Elias, J. E. \& Gygi, S. P. Target-decoy search strategy for mass spectrometrybased proteomics. Methods Mol. Biol. 604, 55-71 (2010).

65. Jabbour, R. E. \& Snyder, A. P. In Biological Identification (ed Schaudies, R. P.) 370-430 (Woodhead Publishing, 2014).

66. Khan, S. J., Abidi, S. N., Tian, Y., Skinner, A. \& Smith-Bolton, R. K. A rapid, gentle and scalable method for dissociation and fluorescent sorting of imaginal disc cells for mRNA sequencing. Fly. (Austin) 10, 73-80 (2016).

67. Deutsch, E. W. et al. The ProteomeXchange consortium in 2017: supporting the cultural change in proteomics public data deposition. Nucleic Acids Res 45, D1100-D1106 (2017).

68. Perez-Riverol, Y. et al. The PRIDE database and related tools and resources in 2019: improving support for quantification data. Nucleic Acids Res 47, D442-D450 (2019)

69. Hendrickson, D. G. et al. Concordant regulation of translation and mRNA abundance for hundreds of targets of a human microRNA. PLoS Biol. 7, e1000238 (2009).

70. Khalil, A. M. et al. Many human large intergenic noncoding RNAs associate with chromatin-modifying complexes and affect gene expression. Proc. Natl Acad. Sci. USA 106, 11667-11672 (2009).

71. Rinn, J. L. et al. Functional demarcation of active and silent chromatin domains in human HOX loci by noncoding RNAs. Cell 129, 1311-1323 (2007).

72. Kohler, S. A., Henderson, B. R. \& Kühn, L. C. Succinate dehydrogenase b mRNA of Drosophila melanogaster has a functional iron-responsive 
element in its 5'-untranslated region. J. Biol. Chem. 270, 30781-30786 (1995).

73. Surdej, P., Richman, L. \& Kühn, L. C. Differential translational regulation of IRE-containing mRNAs in Drosophila melanogaster by endogenous IRP and a constitutive human IRP1 mutant. Insect Biochem Mol. Biol. 38, 891-894 (2008).

\section{Acknowledgements}

The authors thank the Bloomington Drosophila Stock Center at Indiana University, and the Vienna Drosophila Resource Center for sending fly stocks. We thank the labs of Henry Krause and Michael O'Connor for providing fly stocks, and we also thank Andrew Simmonds for sharing vectors and cell culture tools with us. We extend our thanks to Fanis Missirlis and Arash Bashirullah for insightful comments and discussions. K.K.J. wishes to thank the CIHR for supporting this work (MOP 93761). R.L. acknowledges the generous financial support from Deutsche Forschungsgemeinschaft (Koselleck grant LI 415/6), and networking support from the COST Action FeSBioNet (Contract CA15133).

\section{Author contributions}

N.H. co-designed experiments and carried out most experiments, Q.O. conducted the RNAi screen and initial phenotypic characterization, P.C. generated IRP1 transgenic lines and analyzed NOS IR-X phenotypes. R.L. trained Q.O. in iron-related experiments, provided reagents and had an advisory role throughout the project. K.K.J. analyzed the RNA-Seq data, supervised and co-designed experiments, and wrote the manuscript with input from the other authors.

\section{Competing interests}

The authors declare no competing interests.

\section{Additional information}

Supplementary information is available for this paper at https://doi.org/10.1038/s41467019-13237-8.

Correspondence and requests for materials should be addressed to K.K.-J.

Peer review information Nature Communications thanks Hasan Akman, Claudia Blindauer and the other, anonymous, reviewer(s) for their contribution to the peer review of this work. Peer reviewer reports are available.

Reprints and permission information is available at http://www.nature.com/reprints

Publisher's note Springer Nature remains neutral with regard to jurisdictional claims in published maps and institutional affiliations.

\begin{abstract}
(c) $(\mathrm{P}$
Open Access This article is licensed under a Creative Commons Attribution 4.0 International License, which permits use, sharing, adaptation, distribution and reproduction in any medium or format, as long as you give appropriate credit to the original author(s) and the source, provide a link to the Creative Commons license, and indicate if changes were made. The images or other third party material in this article are included in the article's Creative Commons license, unless indicated otherwise in a credit line to the material. If material is not included in the article's Creative Commons license and your intended use is not permitted by statutory regulation or exceeds the permitted use, you will need to obtain permission directly from the copyright holder. To view a copy of this license, visit http://creativecommons.org/licenses/by/4.0/.
\end{abstract}

(C) The Author(s) 2019 